\title{
Effects of Turbulence on Cosmic Ray Propagation in Protostars and Young Star/Disk Systems
}

\author{
Marco Fatuzzo \\ Physics Department, Xavier University, Cincinnati, OH 45207 \\ fatuzzo@xavier.edu \\ and \\ Fred C. Adams \\ Physics Department, University of Michigan, Ann Arbor, MI 48109 \\ fca@umich.edu
}

\begin{abstract}
The magnetic fields associated with young stellar objects are expected to have an hour-glass geometry, i.e., the magnetic field lines are pinched as they thread the equatorial plane surrounding the forming star but merge smoothly onto a background field at large distances. With this field configuration, incoming cosmic rays experience both a funneling effect that acts to enhance the flux impinging on the circumstellar disk and a magnetic mirroring effect that acts to reduce that flux. To leading order, these effects nearly cancel out for simple underlying magnetic field structures. However, the environments surrounding young stellar objects are expected to be highly turbulent. This paper shows how the presence of magnetic field fluctuations affects the process of magnetic mirroring, and thereby changes the flux of cosmic rays striking circumstellar disks. Turbulence has two principle effects: 1) The (single) location of the magnetic mirror point found in the absence of turbulence is replaced with a wide distribution of values. 2) The median of the mirror point distribution moves outward for sufficiently large fluctuation amplitudes (roughly when $\delta B / B_{0}>0.2$ at the location of the turbulence-free mirror point); the distribution becomes significantly non-gaussian in this regime as well. These results may have significant consequences for the ionization fraction of the disk, which in turn dictates the efficiency with which disk material can accrete onto the central object. A similar reduction in cosmic ray flux can occur during the earlier protostellar stages; the decrease in ionization can help alleviate the magnetic braking problem that inhibits disk formation.
\end{abstract}


Subject headings: Cosmic Rays - diffusion - ISM - molecular clouds

\section{Introduction}

Cosmic rays (CRs) significantly influence the physical properties of the interstellar medium and are expected to play an important role in the process of star formation. For example, the cosmic ray flux in star forming regions directly affects the ionization levels (Hayakawa et al. 1961; Spitzer \& Tomasko 1968), heating processes (Glassgold \& Langer 1973), and chemistry (Dalgarno 2006) within the local environment. On scales of $\sim 0.1 \mathrm{pc}$, ionization levels affect the coupling between the gas and the magnetic fields, and in turn, the rate at which star formation occurs (e.g., Fatuzzo et al. 2006; see also the reviews of Shu et al. 1987, McKee \& Ostriker 2007, Crutcher 2012, and references therein). On smaller scales of $\sim 1-100 \mathrm{AU}$, the ionization in circumstellar disks impacts the extent of the disk where the magnetorotational instability mechanism (MRI) remains active, and thereby mediates

accretion rates (Gammie 1996). Understanding how cosmic rays propagate through the highly anisotropic and turbulent star forming environments thus constitutes a fundamental problem in star formation theory.

It is well known that the motion of cosmic rays is strongly affected by the structure of the magnetic field. Specifically, large scale field structures can both focus and mirror charged particles, and a turbulent component that extends to scales smaller than the particle gyration radius results in diffusive motion. All three effects are expected to contribute in star formation environments. Specifically, the gravitational collapse of cores and subsequent formation of protostellar disks are expected to produce hour-glass magnetic field structures in which cosmic rays from the background environment can, on the one hand, get funneled toward the central star/disk object, and, on the other hand, eventually reflect away as they move into a region of increasing magnetic field strength. Note that the magnetic field will attain an hour-glass geometry both in the limit of strong fields, where the collapse is magnetically controlled, and for weak fields, where the collapse flow drags in the field lines. In spite of this ambiguity, observations indicate the presence of hour-glass-like magnetic fields associated with protostars (see, e.g., Davidson et al. 2011) and find alignment between the symmetry axis of the (flattened) protostellar envelopes and the background magnetic fields (Chapmann et al. 2013). In addition, young stellar objects are expected to be highly dynamic and hence drive magnetic turbulence; the goal of this paper is to ascertain how this turbulence affects the propagation of cosmic rays into these systems.

Previous work has considered how cosmic rays propagate through the magnetic field lines that thread molecular cores and related systems (Skilling \& Strong 1976; Cesarsky \& 
Völk 1978; Chandran 2000; Padoan \& Scalo 2005; Desch et al. 2004, Padovani \& Galli 2011), although these analyses did not include the effects of turbulence. The results of this previous work indicate that mirroring tends to dominate over focusing, leading to a net reduction of the cosmic ray ionization rate by a factor of $\sim 2-3$ over most of a solar-mass core with respect to the "background" value for the intercloud medium (outside the core). Additional loss of cosmic ray flux can result from more complicated field configurations due to twisting magnetic field lines that are expected during protostellar collapse (Padovani et al. 2013).

In this paper, we explore the funneling and mirroring effects that occur as cosmic rays move toward the circumstellar disks associated with forming (or newly formed) stars, but also include the effects of magnetic turbulence on cosmic ray propagation. We construct a new nonstandard coordinate system to facilitate the analysis, which lends itself more naturally to the underlying geometry (e.g., by allowing a straightforward implementation of the required condition $\nabla \cdot \mathbf{B}=0)$. As expected, we find that the presence of turbulence leads to a distribution of possible outcomes for essentially equivalent initial conditions. Specifically, there is no longer a simple one-to-one correspondence between the initial conditions of a comic ray and its mirroring point. In the presence of turbulence, and for initial conditions where mirroring occurs far from the disk, cosmic rays are equally likely to penetrate farther inward or reflect earlier - farther out - compared to the turbulence-free mirror radius. In other words, the distribution of mirroring points is symmetric and centered on the value obtained without turbulence. However, for conditions where the mirror points occur in the inner regions near the disk, and for sufficiently large fluctuation amplitudes, turbulence acts primarily to enhance mirroring. As a result, the net effect of turbulence is to increase the efficiency of magnetic mirroring, i.e., turbulence acts to significantly reduce the flux of cosmic rays that reach the circumstellar disk.

This paper is organized as follows. In Section 2, we construct a new coordinate system, including the divergence operator, where one coordinate follows the magnetic lines of the hour-glass-like configuration. The perpendicular coordinates allow us to construct magnetic field perturbations that point in the orthogonal directions and are divergence-free. The specification of the magnetic field perturbations is addressed in Section 3. Next we consider the propagation of cosmic rays, in Section 4, including funneling and mirroring in the absence of turbulence. Section 5 then includes the effects of turbulence on cosmic ray propagation, and presents the results from 120,000 numerical experiments. Finally, we conclude in Section 6 with a summary of our results and a discussion of their implications for star formation and disk accretion. 


\section{Geometry}

This section presents an idealized geometry for the magnetic field extending from a young stellar object. Near the star itself, we expect the field to be dominated by a stellar dipole structure. But the stellar wind will open up the field into a split monopole configuration beyond some radius that is much larger than the stellar radius, and much smaller than the radius of the circumstellar disk. We therefore model the unperturbed (static) magnetic field extending from the stellar system with a split-monopole component that eventually merges with a uniform "background" field $\mathbf{B}_{\infty}=B_{\infty} \hat{z}$. This idealized form is expected to adequately capture the important aspects of the underlying magnetic field structure for the $z>0$ hemisphere beyond an inner boundary, which we define through a radius $R$ (we specify the value below). Note that incident cosmic rays that cross the inner boundary are expected to have a high probability of interacting with the circumstellar disk material. The resulting hour-glass-like geometry (for positive $z$ ) is then conveniently given by the expression

$$
\mathbf{B}_{\mathbf{0}}=B_{\infty}\left[\frac{\varepsilon}{\xi^{2}} \hat{r}+\hat{z}\right],
$$

where $\xi=r / R$. The value of $\varepsilon$ defines the relative strength of the split-monopole component with respect to $B_{\infty}$, and determines the approximate crossover radius $\xi_{c}=\sqrt{\varepsilon}$ between the 'nearly radial' and 'nearly uniform' regions of the magnetic field.

With this configuration, the magnetic field is current-free and curl-free, and can be written as the gradient of a scalar field. We define a scalar field $p$ that serves as the first field of the coordinate system, i.e.,

$$
p=\xi \cos \theta-\frac{\varepsilon}{\xi},
$$

where the gradient $\nabla p$ defines a vector field that points in the direction of the magnetic field. We can then construct the perpendicular vector field $\nabla q$ from a second scalar field $q$ of the coordinate system, i.e.,

$$
q=\frac{1}{2} \xi^{2} \sin ^{2} \theta-\varepsilon \cos \theta
$$

The pair $(p, q)$ thus represents a set of perpendicular coordinates in the poloidal plane, with the azimuthal angle $\phi$ providing the third scalar field of the coordinate system. We note

that the value of $q$ remains constant on a given field line Adams 2011; Adams \& Gregory 2012 .

The dimensionless covariant basis vectors $\underline{\epsilon}_{j}$ are given by the usual relations

$$
\underline{\epsilon}_{p}=\nabla p, \quad \underline{\epsilon}_{q}=\nabla q, \quad \text { and } \quad \underline{\epsilon}_{\phi}=\nabla \phi,
$$


where the gradient is written in terms of the variables $(\xi, \theta, \phi)$. Evaluating these quantities, we obtain

$$
\begin{gathered}
\underline{\epsilon}_{p}=\left(\cos \theta+\frac{\varepsilon}{\xi^{2}}\right) \hat{r}-\sin \theta \hat{\theta}=\frac{\varepsilon}{\xi^{2}} \hat{r}+\hat{z}=\frac{\mathbf{B}_{\mathbf{0}}}{B_{\infty}} \\
\underline{\epsilon}_{q}=\xi \sin \theta\left[\sin \theta \hat{r}+\left(\cos \theta+\frac{\varepsilon}{\xi^{2}}\right) \hat{\theta}\right]
\end{gathered}
$$

and

$$
\underline{\epsilon}_{\phi}=\frac{1}{\xi \sin \theta} \hat{\phi}
$$

We note that the quantities $\underline{\epsilon}_{j}$ are basis vectors, rather than unit vectors, so that their length are not, in general, equal to unity. The corresponding unit vectors can trivially be written as

$$
\hat{n}_{j}=h_{j} \underline{\epsilon}_{j}
$$

where the corresponding scale factors $h_{j}=1 /\left|\underline{\epsilon}_{j}\right|$ are given by

$$
\begin{aligned}
& h_{p}=\left[1+2 \cos \theta \frac{\varepsilon}{\xi^{2}}+\frac{\varepsilon^{2}}{\xi^{4}}\right]^{-1 / 2}=\frac{B_{\infty}}{B_{0}}, \\
& h_{q}=\frac{1}{\xi \sin \theta}\left[1+2 \cos \theta \frac{\varepsilon}{\xi^{2}}+\frac{\varepsilon^{2}}{\xi^{4}}\right]^{-1 / 2},
\end{aligned}
$$

and

$$
h_{\phi}=\xi \sin \theta \text {. }
$$

In the limit of large $\xi$, the field lines point in the $\hat{z}$ direction. The field lines that emanate radially outward from the origin $(\xi=0)$ with angle $\theta_{0}$ thus map onto a cylinder at large (spherical radii) $\xi$ with cylindrical radius $\varpi_{\infty}$. This radius is determined by the condition $q$ $=$ constant, i.e.,

$$
\frac{1}{2} \xi^{2} \sin ^{2} \theta-\varepsilon \cos \theta=-\varepsilon \cos \theta_{0}
$$

which can be rewritten in the form

$$
\varpi_{\infty}^{2}=2 \varepsilon R^{2}\left(1-\cos \theta_{0}\right)
$$

where $\cos \theta_{\infty} \rightarrow 1$. The outermost radius $\varpi_{\max }$ occurs for $\cos \theta_{0}=0$, i.e., the magnetic field line that leaves from the equator of the central region, and the effective feeding radius of the system is thus given by

$$
\varpi_{\max }=(2 \varepsilon)^{1 / 2} R
$$

In order to relate the $(p, q, \phi)$ coordinate system with the more traditional cartesian coordinate system, we show several field lines in Figure 1 (solid curves) for the case that 
$\varepsilon=10^{4}$. "Equipotential" lines of constant $p$ are also shown (dotted curves). We note that requiring $z \geq 0$ limits the coordinate $q$ to the range $-\varepsilon \leq q \leq 0$. Furthermore, in the limit that $\varepsilon \gg 1$, the inner boundary $\xi=1$ is well approximated by the $p=-\varepsilon$ line.

\section{The Turbulent Magnetic Field}

At present, a complete theory of MHD turbulence in the interstellar medium remains elusive. Nevertheless, it is generally understood that turbulence is driven from a cascade of longer wavelengths to shorter wavelengths as a result of wave-wave interactions. For strong MHD turbulence in a uniform medium, this cascade seemingly produces eddies on small spatial scales that are elongated in the direction of the underlying magnetic field, so that the components of the wave vector $k_{\perp}$ and $k_{\|}$are related by the expression $k_{\|} \propto k_{\perp}^{2 / 3}$ (Sridhar \& Goldreich 1994; Goldreich \& Sridhar 1995; Cho \& Lazarian 2003). It is beyond the scope of this paper to extend these results for our non-uniform geometry. Since our aim here is to determine the possible effects of turbulence on cosmic ray propagation into a star/disk system, we will assume a reasonable form for the turbulent magnetic field as guided by basic principles.

Following the standard numerical approach for analyzing the fundamental physics of ionic motion in a turbulent magnetic field, we treat the total magnetic field $\mathbf{B}$ as a spatially turbulent component $\delta \mathbf{B}$ superimposed onto the static hour-glass-like background field $\mathbf{B}_{\mathbf{0}}$ described in Section 2. The turbulent field $\delta \mathbf{B}$ is generated by summing over a large number of randomly polarized waves with effective wave vectors $\bar{k}_{n}$ logarithmically spaced between $\bar{k}_{\min }$ and $\bar{k}_{\max }$ (e.g., Giacalone \& Jokipii 1994; Casse et al. 2002; O'Sullivan et al. 2009; Fatuzzo et al. 2010). We assume that each term of the turbulent field is Alfvénic in the sense that $\delta \mathbf{B}_{\mathbf{n}} \perp \mathbf{B}_{\mathbf{0}}$, and satisfies the no-monopole condition $\nabla \cdot \delta \mathbf{B}_{\mathbf{n}}=0$. Since the Alfvén speed $v_{A}$ is much less than that of the relativistic cosmic rays, we can adopt a static turbulent field for calculating the effects on particle motion. This simplification then removes the necessity of specifying a dispersion relation for each term.

Given these considerations, we assume a turbulent field of the form

$$
\delta \mathbf{B}=\sum_{n=1}^{N} A_{n}(p, q) \cos \left(\bar{k}_{n} p+\beta_{n}\right)\left[\cos \alpha_{n} \hat{n}_{q}+\sin \alpha_{n} \hat{n}_{\phi}\right],
$$

where the direction and phase of each term is set through a random choice of $\alpha_{n}$ and $\beta_{n}$. The values of $\bar{k}_{1}=\bar{k}_{\text {min }}$ and $\bar{k}_{N}=\bar{k}_{\text {max }}$ are defined in terms of a maximum and minimum wavelength, as defined by the condition that $\bar{k}_{1} p$ and $\bar{k}_{N} p$ advance by $2 \pi$ as the distance along a field line (as defined by its value of $q$ ) from the inner boundary (i.e., $\xi=1$ ) advances by $\lambda_{\max }$ 


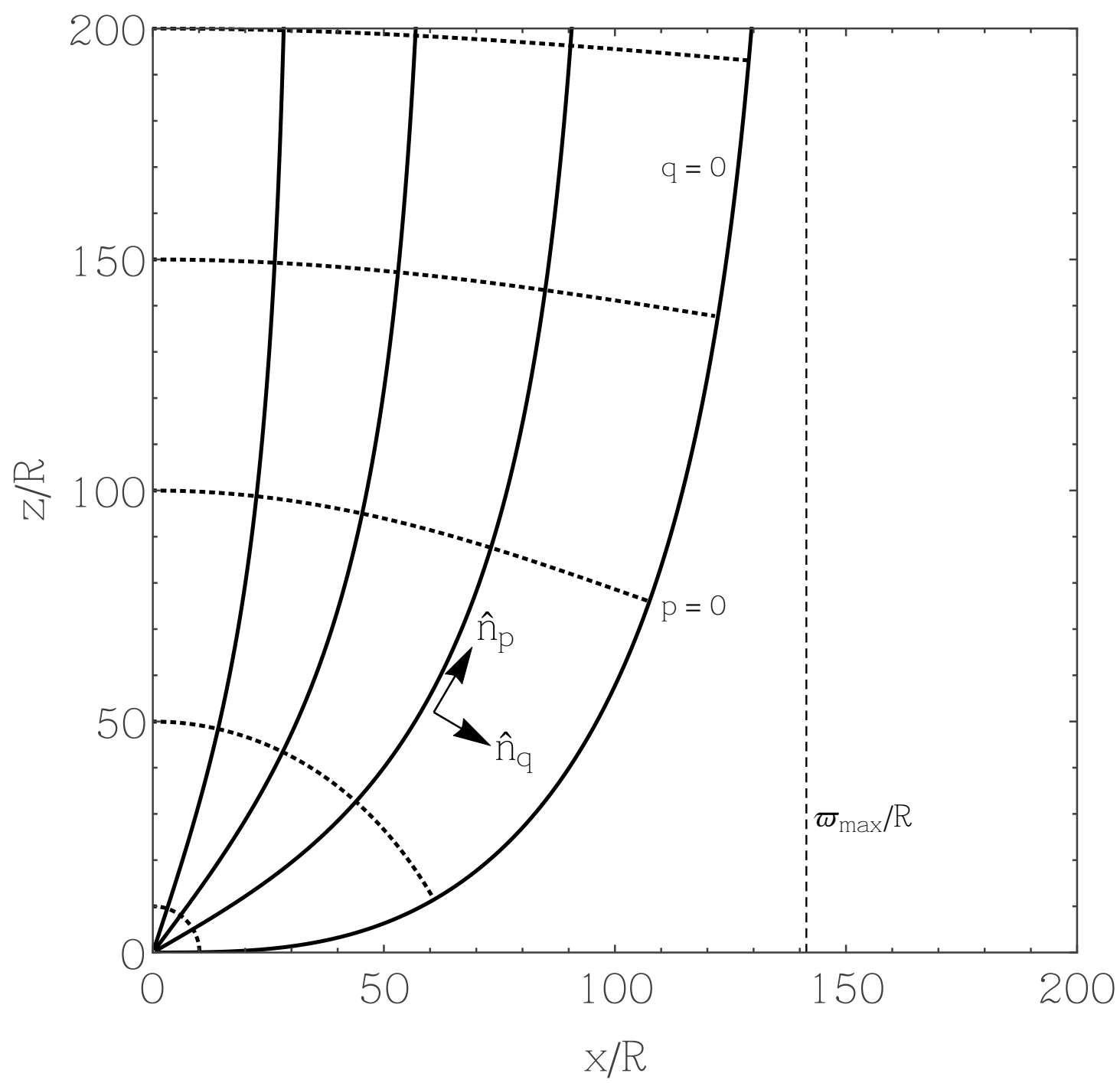

Fig. 1.- Magnetic field geometry for $\varepsilon=10^{4}$. Solid lines denote magnetic fields line, for which $q$ is constant. The corresponding values of $q$ are, from the inner most line to the outer most line, given by: $q=-9500,-8000,-5000$ and 0 . Dotted lines denote "equipotentials" on which $p$ is constant. Moving outward, the coordinate has values $p=-990,-150,0,83$ $1 / 3$, and 150 . The dashed line represents the cylindrical outer boundary at $\varpi_{\max } / R$. Note that the unit vectors $\hat{n}_{p}$ and $\hat{n}_{q}$ are not drawn to scale. 
and $\lambda_{\text {min }}$, respectively. All other values of $k_{n}$ are then found through an even logarithmic binning, with the total number of terms in the sum given by $N=N_{k} \log _{10}\left[\lambda_{\max } / \lambda_{\min }\right]$, where $N_{k}$ is the number of waves desired per decade. Following the results of previous studies (Fatuzzo et al. 2010; see also Everett \& Zweibel 2011), we set the number of waves per decade to $N_{k}=25$.

To illustrate how the resulting turbulent field will appear, we first note that the values of $\bar{k}_{1}$ and $\bar{k}_{N}$ are, to a high level of approximation, independent of the field line being considered when $\lambda_{\max } \ll \sqrt{\varepsilon} R$ and $\varepsilon \gg 1$. The first condition ensures that the field lines remain very nearly radial as one follows a field line outward from then inner surface a distance of one wavelength $\lambda_{0}$. In so doing, the value of $\xi$ therefore changes from 1 to $1+\lambda_{0} / R$, and the corresponding change in $p$ is then given by

$$
\Delta p=\frac{\lambda_{0}}{R}\left[\cos \theta+\frac{\varepsilon R}{\lambda_{0}+R}\right] \approx \frac{\varepsilon \lambda_{0}}{\lambda_{0}+R},
$$

where the conditions $\lambda_{0} \ll \sqrt{\varepsilon} R$ and $\varepsilon \gg 1$ allow us to ignore the $\cos \theta$ term in the final expression. Since one wavelength corresponds to a change in the argument $\bar{k} p$ of $2 \pi$, one then finds

$$
\bar{k} \approx \frac{2 \pi\left(\lambda_{0}+R\right)}{\varepsilon \lambda_{0}}
$$

To demonstrate how the wave profile changes along a field line, we plot the function $g_{q}[p(\xi)]=$ $\cos [\bar{k} p(\xi)]$ in Figure 2 for a wavelength of $\lambda_{0}=0.1 R$, where $p$ is evaluated as a function of $\xi$ for a fixed value of $q$ (i.e., for a specified field line). We present results for the limiting values $q=-\varepsilon$ and $q=0$. Clearly, the "wave-like" nature of the turbulence, as defined by equation (15), is the nearly same for all field lines near the inner surface.

Likewise, the function $g_{q}[p(\xi)] \approx \cos [\bar{k} z / R]$ when $\xi \gg \xi_{c}=\sqrt{\varepsilon}$, so that the wave-like nature of the turbulence is the same for all field lines beyond the crossover radius $\xi_{c}$. The wavelength $\lambda_{\infty}$ in this region is then related to the wavelength at the inner boundary through the expression

$$
\frac{\lambda_{\infty}}{R}=\frac{\varepsilon \lambda_{0}}{\lambda_{0}+R}
$$

In the cross-over region, we can characterize the wavelength $\lambda$ of the turbulence associated with the inner boundary wavelength $\lambda_{0}$ through the condition that $\bar{k} p$ changes by $2 \pi$ as the radius changes from $\xi$ to $\xi+\lambda / R$ along the $q=-\varepsilon$ field line (so that $p=\xi-\varepsilon / \xi$ ). The results are shown in Figure 3 for the values of $\lambda_{0}=0.1 R$ and $\lambda_{0}=10^{-5} R$. As a point of reference, we also plot the radius of gyration for a proton with Lorentz factor $\gamma=10^{2}$ moving perpendicular to the background field $\mathbf{B}_{\mathbf{0}}$, as given by the expression

$$
R_{g}=\gamma \frac{m_{p} c^{2}}{e B_{0}}
$$




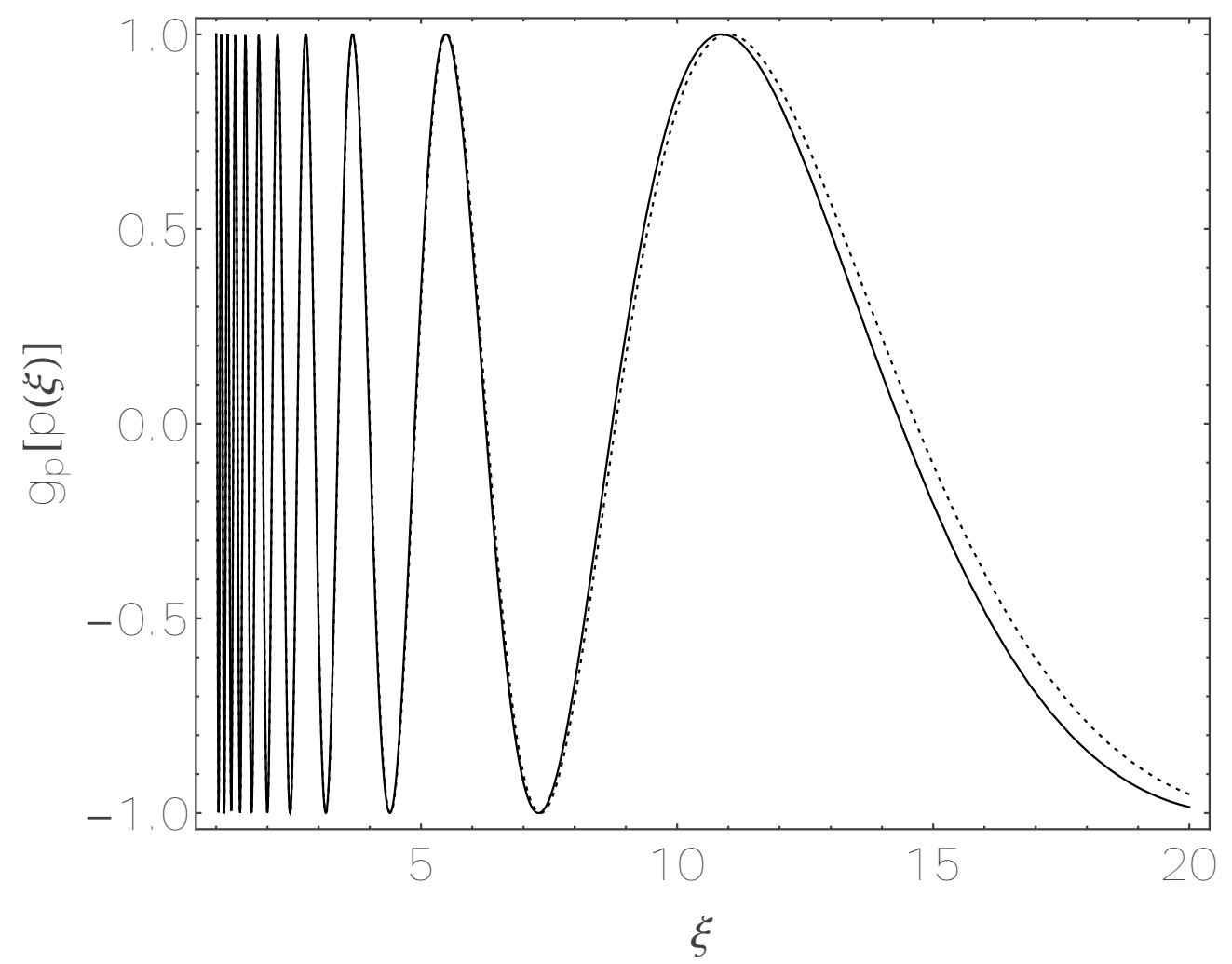

Fig. 2.- The function $g_{q}[p(\xi)]=\cos [\bar{k} p(\xi)]$ for the case $\lambda=0.1 R$, with the solid curve representing the case $q=-\varepsilon$ and the dashed curve representing the case $q=0$. 
where we have assumed representative values of $B_{\infty}=25 \mu \mathrm{G}$ and $R=2 \times 10^{12} \mathrm{~cm}$ (see discussion below). Note that for such a particle, the radius of gyration always falls within the range of wavelengths spanning the turbulence profile generated by setting $\lambda_{\max }=0.1 R$ and $\lambda_{\min }=10^{-5} R$.

To complete the analysis, we note that since the turbulent field is axisymmetric, the divergence operator in our $(p, q, \phi)$ coordinate system takes the form

$$
\nabla \cdot \delta \mathbf{B}_{\mathbf{n}}=\frac{\cos \left(\bar{k}_{n} p+\beta_{n}\right) \cos \left(\alpha_{n}\right)}{h_{p} h_{q} h_{\phi}}\left[\frac{\partial}{\partial q}\left(h_{p} h_{\phi} A_{n}\right)\right]=0
$$

which requires

$$
h_{p} h_{\phi} A_{n}=f(p, \phi) .
$$

Noting that $h_{\phi}=\xi \sin \theta$ and $h_{p}=B_{\infty} / B_{0}$, and setting $f(p)$ equal to the constant $A_{0 ; n}$, one then finds

$$
A_{n}(\xi, \theta)=\frac{A_{0 ; n}}{\xi \sin \theta} \frac{B_{0}}{B_{\infty}},
$$

where the desired spectrum of the turbulent magnetic field is set through the appropriate choice of scaling for an assumed turbulent profile, i.e.,

$$
A_{0 ; n}^{2}=A_{0 ; 1}^{2}\left[\frac{k_{n}}{k_{1}}\right]^{-\Gamma} \frac{\Delta k_{n}}{\Delta k_{1}}=A_{0 ; 1}^{2}\left[\frac{k_{n}}{k_{1}}\right]^{-\Gamma+1}
$$

where, e.g., $\Gamma=3 / 2$ for Kraichnan and $\Gamma=5 / 3$ for Kolmogorov turbulence. We note that for our logarithmic binning scheme, the value of $\Delta k_{n} / k_{n}$ is the same for all values of $n$. The value of $A_{0 ; 1}$ is set by an amplitude parameter $\eta$ that specifies the average energy density of the turbulent field with respect to the background hour-glass field at the inner boundary; specifically, $\eta$ is defined through the expression

$$
\eta=\frac{\left\langle\delta B^{2}\right\rangle}{B_{0 s}^{2}}
$$

where $B_{0 s}$ is the magnitude of the hour-glass magnetic field at $\xi=1$.

Figure 4 presents four different turbulent field lines produced using four different values of $\eta$. Moving outward, the amplitude parameter $\eta=30$ for the $q=-9,500$ field line, $\eta=10$ for the $q=-8000$ field line, $\eta=3$ for the $q=-5000$ field line, and $\eta=1$ for the $q=0$ field line. Note that for $\xi \ll \xi_{c}$, the "nearly radial" region of the background field, the magnitude of the turbulent field scales as $\delta B \sim \sqrt{\eta} B_{0} / \xi \sim \sqrt{\eta} B_{\infty} \varepsilon / \xi^{3}$. On the other hand, for $\xi \gg \xi_{c}$, the "nearly uniform" region of the background field, the magnitude of the turbulent field scales as $\delta B \sim \sqrt{\eta} B_{\infty} R / \varpi_{\infty}$. 


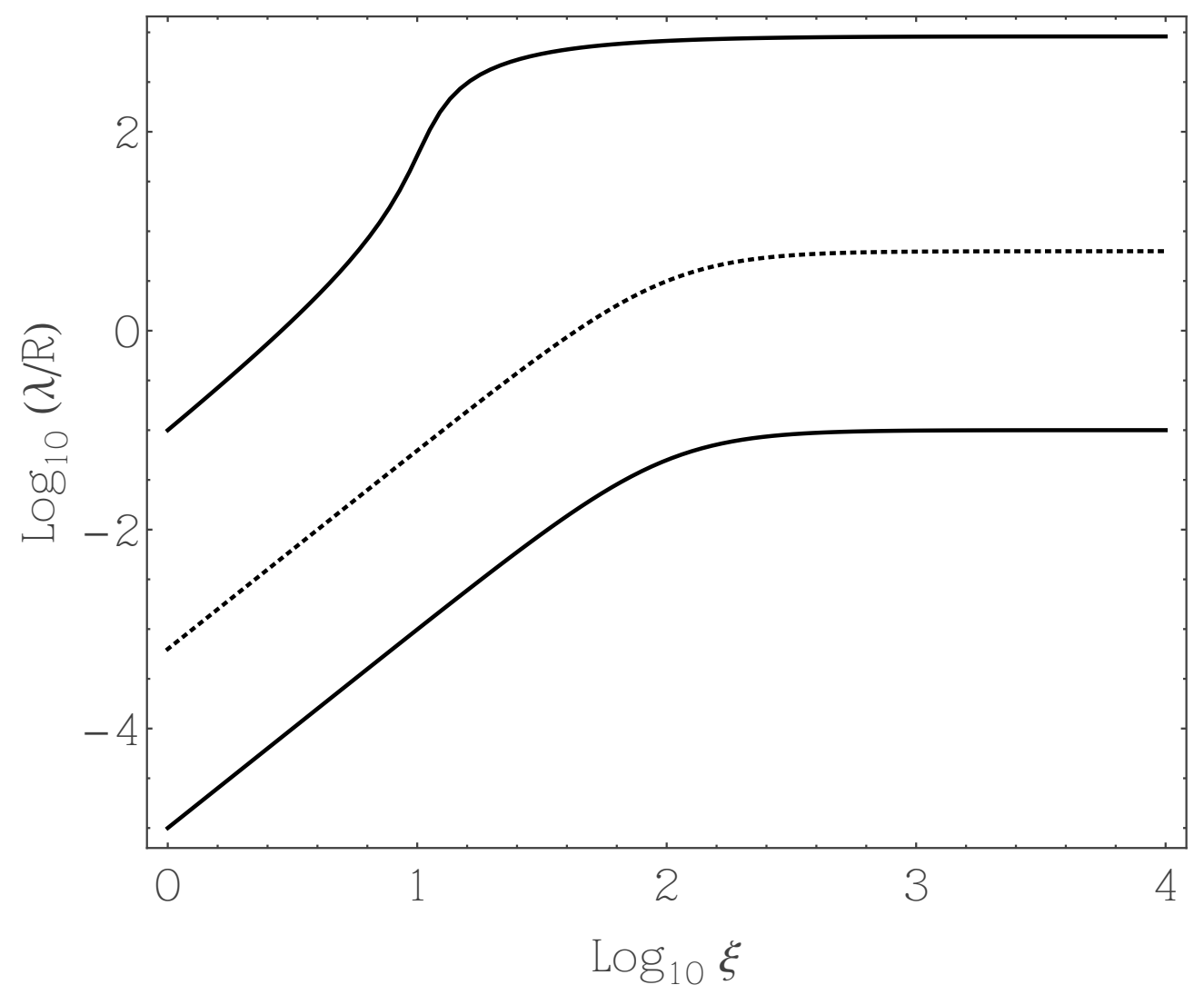

Fig. 3.- The values of $\lambda$ as a function of $\xi$, as defined in the text, associated with $\lambda_{0}=0.1 R$ (upper solid curve) and $\lambda_{0}=10^{-5} R$ (lower solid curve). As a point of reference, we also plot the value of $R_{g} / R$ as a function of $\xi$ (dotted curve), where $R_{g}$ is the radius of gyration for a proton with Lorentz factor $\gamma=10^{2}$ moving perpendicular to the $q=-\varepsilon$ field line, for the assumed representative values of $\varepsilon=10^{4}, B_{\infty}=25 \mu \mathrm{G}$, and $R=2 \times 10^{12} \mathrm{~cm}$. 


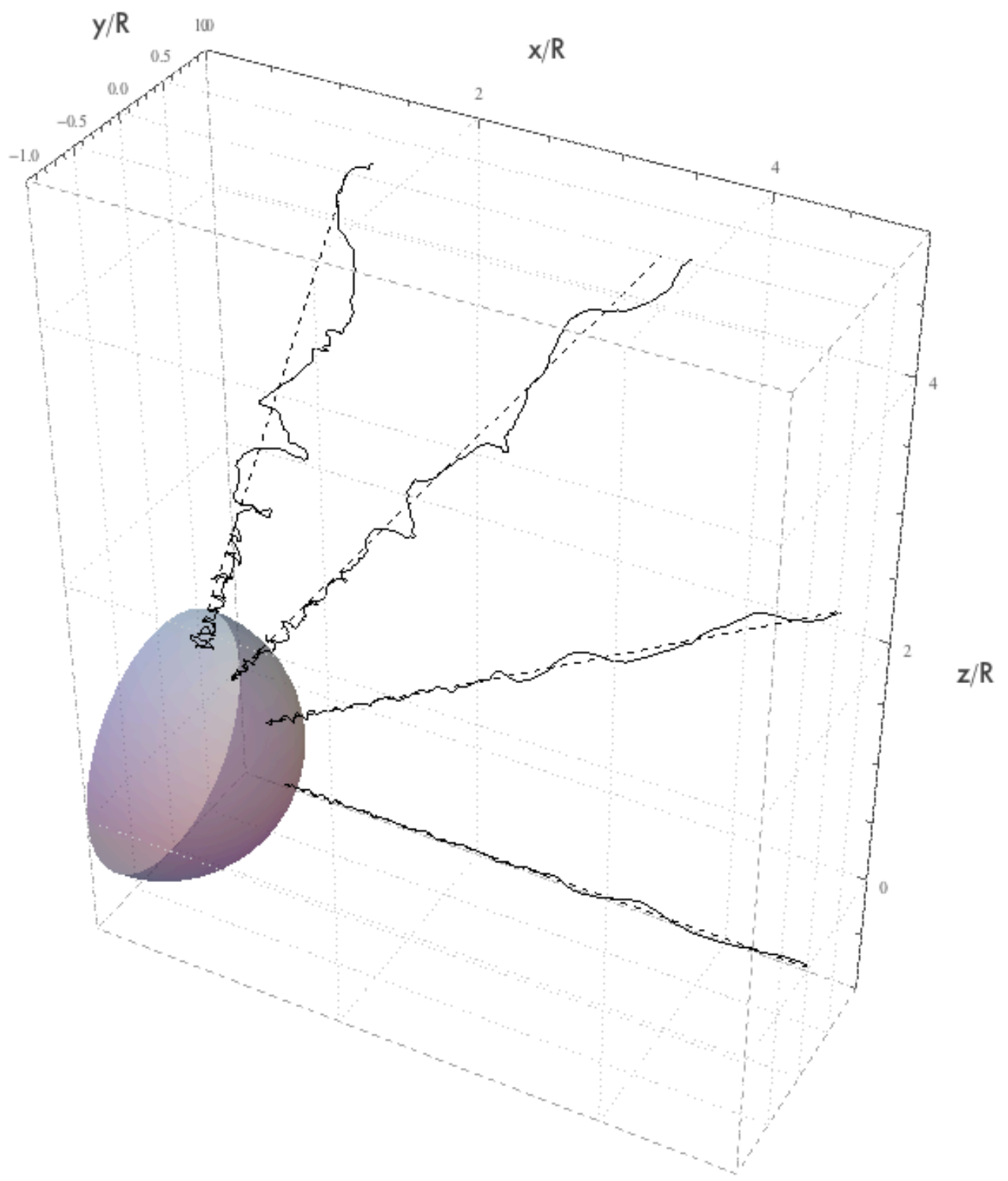

Fig. 4.- Four different turbulent field lines produced using different values of the amplitude parameter $\eta$. Moving outward, $\eta=30$ for the $q=-9$, 500 field line, $\eta=10$ for the $q=-8000$ field line, $\eta=3$ for the $q=-5000$ field line, and $\eta=1$ for the $q=0$ field line. The gray surface denotes the inner boundary. 


\section{Basic Cosmic Ray Dynamics}

For clarity, we consider first the motion of relativistic charged protons through the background hour-glass magnetic field without a turbulent component (Padovani \& Galli 2011). The general equations that govern the motion of protons with Lorentz factor $\gamma$ through a magnetic field are

$$
\frac{d}{d t}\left(\gamma m_{p} \mathbf{v}\right)=\frac{e \mathbf{v} \times \mathbf{B}}{c} \quad \text { and } \quad \frac{d \mathbf{r}}{d t}=\mathbf{v},
$$

and are readily solved using standard numerical methods.

The magnetic moment of a relativistic proton is given by

$$
\mu=\frac{\gamma^{2} m_{p} v_{\perp}^{2}}{2 B}
$$

and is an adiabatic invariant under the condition that the field does not change significantly within a cyclotron radius, i.e., in the limit

$$
\frac{\gamma m_{p} c v_{\perp}}{e B} \ll \frac{B}{|\nabla B|},
$$

where $v_{\perp}$ is the component of the proton velocity perpendicular to the magnetic field through which it is moving. For the magnetic field configuration used here, this limit is most stringent at the inner surface, where it can be expressed in terms of a critical Lorentz factor

$$
\gamma \ll \gamma_{c r i t} \equiv \frac{e}{m_{p} c^{2}}\left[\frac{B^{2}}{|\nabla B|}\right]_{R}=\frac{\varepsilon}{2} \frac{e B_{\infty} R}{m_{p} c^{2}} .
$$

Since the Lorentz factor of the protons remains constant in a time-independent magnetic field, the adiabatic invariance can be expressed as

$$
\frac{\sin ^{2} \alpha_{p}}{B}=\text { constant }
$$

where $\alpha_{p}$ is the pitch angle of a cosmic ray at a location where the field strength is $B$. As a cosmic ray moves toward the inner radius, its pitch angle must increase to match the increasing field strength; however, since $\sin \alpha_{p} \leq 1$, the cosmic ray must eventually reflect at a mirror point in the field. For field structures with $\varepsilon \gg 1$, cosmic rays initially far from the cross-over region $\left(\xi \gg \xi_{c}\right)$ must therefore have pitch angles less than a maximum value if they are to penetrate the inner boundary at $\xi=1$; this condition takes the form

$$
\alpha_{p}<\alpha_{c r i t}=\sqrt{\frac{B_{\infty}}{B_{0}(R)}}=\frac{1}{\sqrt{\varepsilon}} .
$$


In Figure 5, we plot the mirror point location (radius) as a function of the cosine of the injection pitch angle for cosmic rays injected into an hour-glass-like field with $\varepsilon=10^{4}$. The figure shows results for three different field lines (as defined by their corresponding values of $q$ ), where all cases start from an initial value of $z_{i}=10^{3} R$.

Figure 6 then shows the trajectories of three protons, each injected at $z_{i}=10^{3} R$ with Lorentz factor $\gamma=10^{2}$, into an hour-glass field with $R=2 \times 10^{12} \mathrm{~cm}, B_{\infty}=25 \mu \mathrm{G}$, and $\varepsilon=10^{4}$; the three cases correspond to the parameter choices: 1) $\cos \alpha_{p}=0.98$ and $q=-9500,2) \cos \alpha_{p}=0.99$ and $q=-5000$, and 3) $\cos \alpha_{p}=0.995$ and $q=0$. The mirror radii, as shown in this figure, are in excellent agreement with the expected values as illustrated in Figure 5. We note that for the chosen field parameters, $\gamma \ll \gamma_{\text {crit }}=8 \times 10^{4}$.

If we assume that the velocity distribution of cosmic rays is isotropic at distances much greater than $\xi_{c}$, then the fraction $\mathcal{F}$ of the cosmic ray flux that penetrates to the inner surface $(\xi=1)$ is given by

$$
\mathcal{F}=1-\cos \alpha_{\text {crit }}=1-\left[1-\varepsilon^{-1}\right]^{1 / 2} \approx \frac{1}{2 \varepsilon} .
$$

Clearly, only a small fraction of cosmic rays penetrate all the way to the depth $\xi=1$, with the remainder being mirrored back. However, this apparent reduction in the cosmic ray flux impinging upon the stellar disk is offset by the funnel effect resulting from the hour-glass geometry. In the limit of large $\xi$, the field lines point in the $\hat{z}$ direction; as a result, field lines that cross the inner boundary (at $\xi=1$ ) can be mapped onto a cylinder at large $\xi$. Using the effective feeding radius of the system, as given by equation (14), the effective input area $A_{\text {eff }}$ of the system (the area from which cosmic rays are harvested from the background medium) is thus given by

$$
A_{\mathrm{eff}}=2 \pi \varepsilon R^{2} .
$$

The cosmic rays are thus funneled from an initial area $A_{\text {eff }}$ to an inner region with crosssectional area $A_{s}=\pi R^{2}$, which enhances the cosmic ray flux by a factor $\mathcal{E}=2 \varepsilon$. The net factor by which the cosmic ray flux changes is thus given by

$$
f=\mathcal{F E}=1 .
$$

In other words, to leading order, the mirror effect and the funnel effect cancel out (in agreement with previous treatments, e.g., Padovani \& Galli 2011). We note that a simple fluxfreezing argument gives a similar cancellation between the mirror effect and the funnel effect.

\section{Effects of Turbulence}

This section generalizes the calculation of the previous section to include turbulent fluctuations of the magnetic field, and shows how cosmic ray propagation can be affected. In 


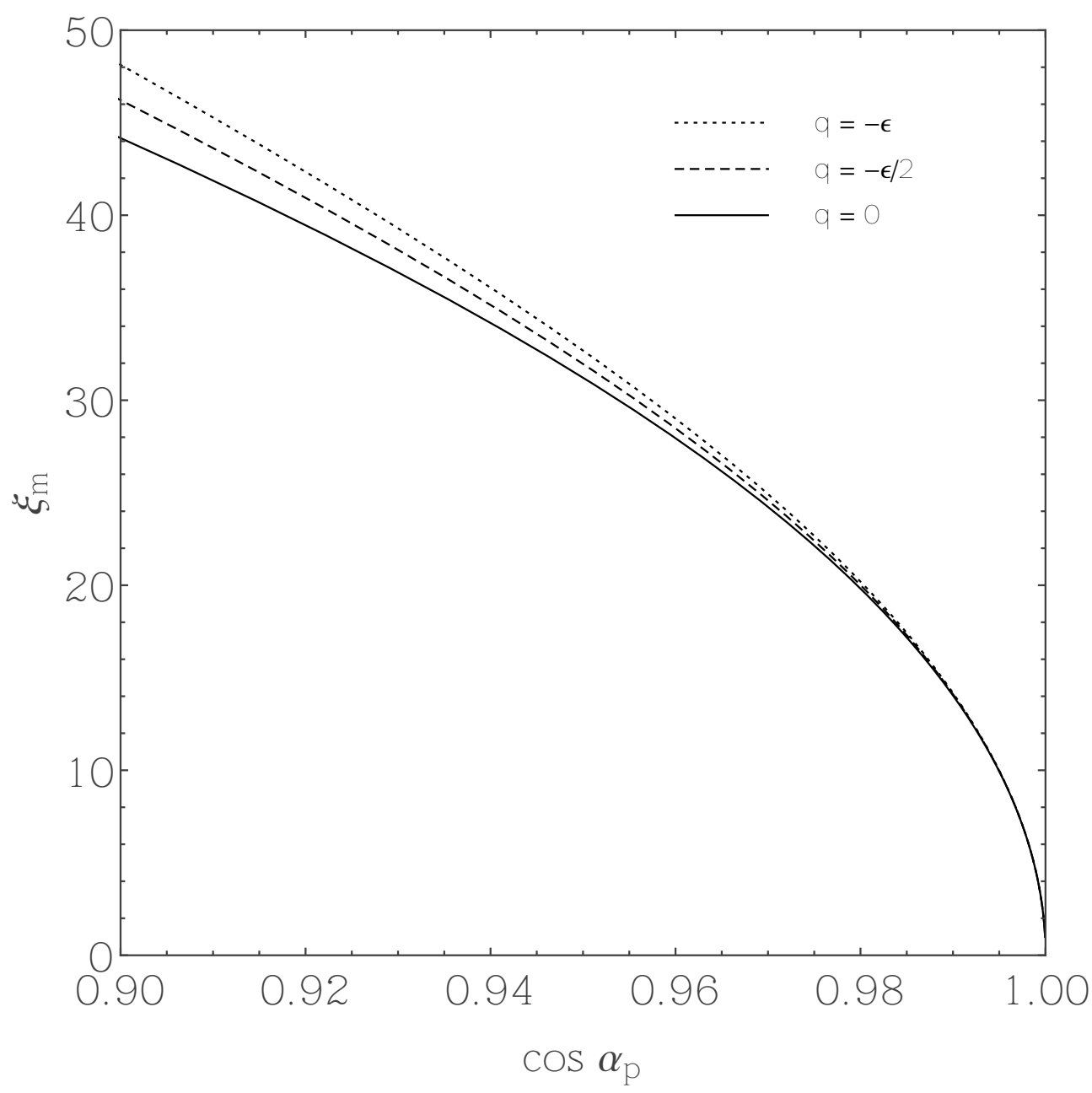

Fig. 5.- The mirror radius $\xi_{m}$ as a function of the cosine of the injection inclination angle for comic-rays injected into an hour-glass field with $\varepsilon=10^{4}$ along three different field lines corresponding to $q=0$ (solid curve), $q=-\varepsilon / 2$ (dashed curve) and $q=-\varepsilon$ (dotted curve); all trajectories start from an initial position with $z_{i}=10^{3} R$. 


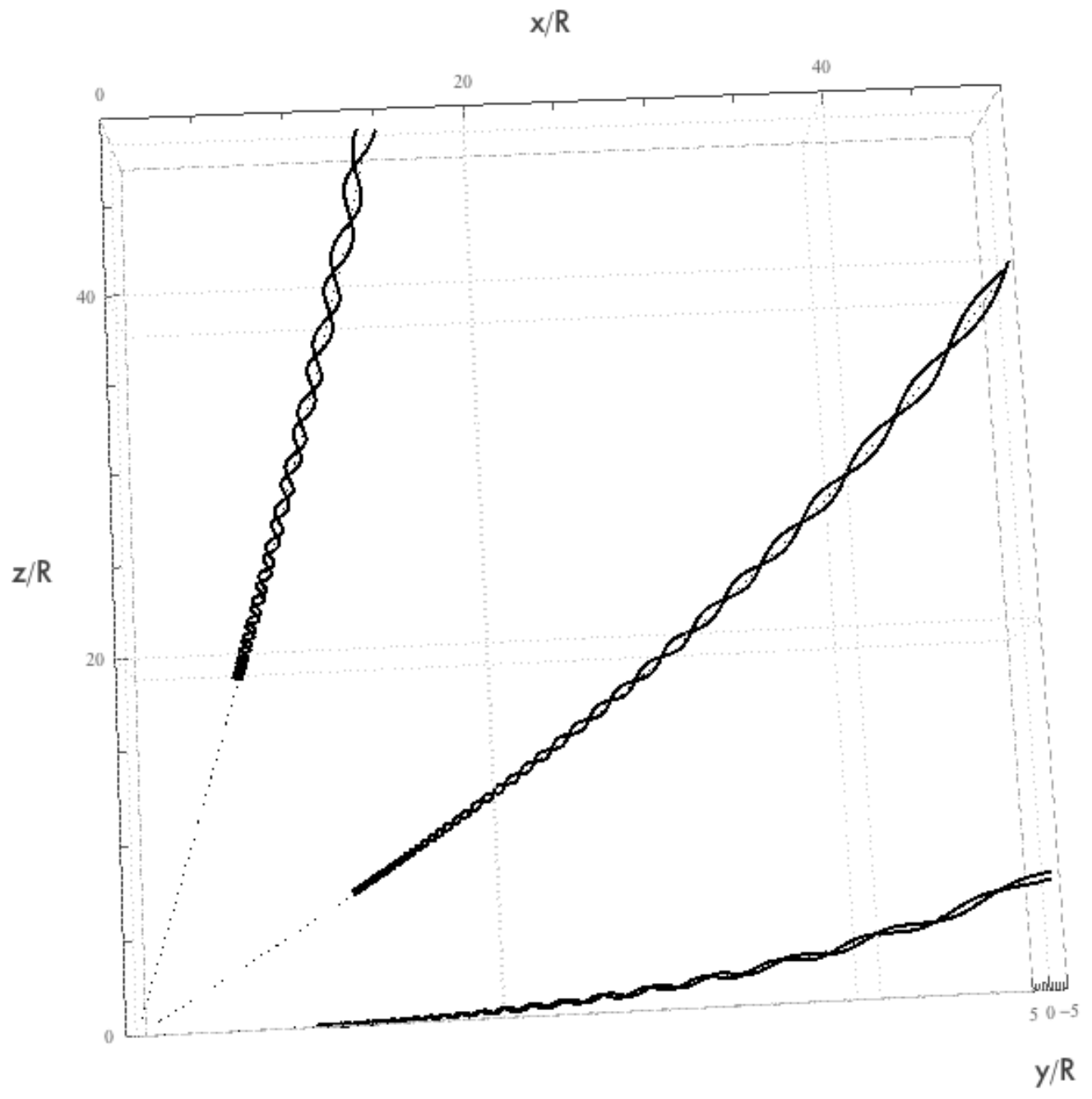

Fig. 6. - Particle trajectories for three protons injected at $z_{i}=10^{4} R$ with Lorentz factors $\gamma=10^{2}$ into an hour-glass field with $R=2 \times 10^{12} \mathrm{~cm}, B_{\infty}=25 \mu \mathrm{G}$, and $\varepsilon=10^{4}$. Dashed lines show the magnetic field lines on which the particles were injected. Left most trajectory: $q=-9500, \cos \alpha_{p}=0.98$. Center trajectory: $q=-5000, \cos \alpha_{p}=0.99$. Right most trajectory: $q=0, \cos \alpha_{p}=0.995$. 
the presence of turbulence, charged particles interact resonantly with the magnetic field, and are most strongly influenced by field fluctuations with wavelength $\lambda \sim R_{g}$. As a result, the magnetic moment of a cosmic ray is no longer invariant. This point is illustrated in Figure 7, which plots the product $\left(B_{\infty} / B\right) \sin ^{2} \alpha$ as a function of $\xi$ for a proton injected toward the inner boundary on the $q=-5000$ field line with a pitch angle defined by $\cos \alpha_{p}=0.99$; results are shown for four different turbulence levels, with amplitude parameter $\eta=0,0.1$, 1 , and 10. As expected, the presence of magnetic turbulence can displace the location at which mirroring occurs.

Although the governing equations are deterministic, the motion of charged particles through a turbulent magnetic field is chaotic in nature. As a result, a complete analysis requires a statistical approach. Toward that end, we have carried out a large ensemble of numerical experiments to study cosmic ray propagation through a turbulent magnetic field. We define a single experiment as a numerical investigation of the particle dynamics through a given type of turbulent environment, starting with a given particle injection scenario, as described below.

For all experiments, we adopt fiducial values of $B_{\infty}=25 \mu \mathrm{G}, R=2 \times 10^{12} \mathrm{~cm}$, and $\varepsilon=10^{4}$ for the background magnetic field, and we use $\lambda_{\max }=0.1 R$, and $\lambda_{\min }=10^{-5} R$ for the turbulent field. We focus primarily on Kolmogorov turbulence $(\Gamma=5 / 3)$, but perform an experiment using Kraichnan turbulence $(\Gamma=3 / 2)$ for comparison. The turbulence level, as defined by $\eta$, is one of the experimental parameters. Particles are injected toward the origin from $z_{i}=10^{3} R$ with one of the following injection scenarios: 1) all particles are injected with the same inclination angle $\alpha_{p}$ and from the same field line, as specified by $\left.q ; 2\right)$ all particles are injected with the same inclination angle $\alpha_{p}$, but randomly distributed throughout the portion of the $x-y$ plane (at fixed height $z=z_{i}$ ) that funnels particles directly to the inner surface; 3 ) same as scenario 2 , but with particles injected with inclination angles drawn randomly from a flat (uniform) $\mu_{p}=\cos \alpha_{p}$ distribution between 0.9999 and 1. For each experiment, we numerically integrate the equations of motion for $N_{p}=10^{4}$ monoenergetic protons (as defined by their Lorentz factor $\gamma$ ) until reflection occurs, with each particle sampling its own unique realization of the magnetic turbulence through a random selection of the values for $\alpha_{n}$ and $\beta_{n}$. The radius $\xi_{m}$ where mirroring occurs is the output of each particle run, and the median value $\bar{\xi}_{m}$ and normalized width $\sigma_{\xi}$ of the ensuing distributions then serve as the output measures for a given experiment. The experiments and corresponding output measures are summarized in Table 1, and the distributions of mirroring radii for each experiment are shown in Figures $8-19$. Note that the values of $\bar{\xi}_{m}$ are given in terms of the median for the corresponding distribution one would obtain in the absence of turbulence, which is denoted as $\bar{\xi}_{m 0}$, whereas the values of $\sigma_{\xi}$ are given in terms of $\bar{\xi}_{m}$. 


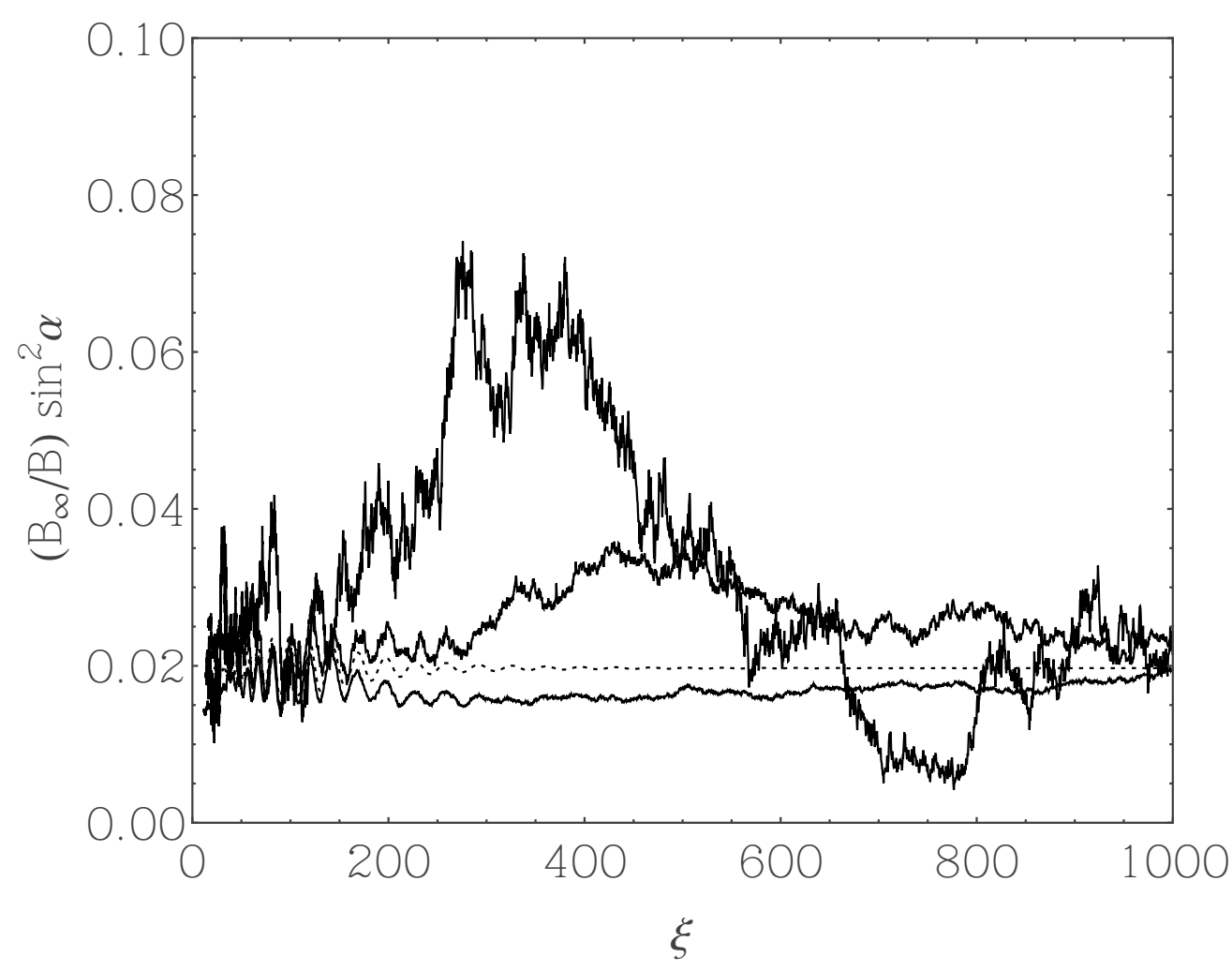

Fig. 7.- The value of $\left(B_{\infty} / B\right) \sin ^{2} \alpha$ as a function of $\xi$ for a protons injected on the $q=-5000$ field line toward the inner boundary with an initial pitch angle corresponding to $\cos \alpha_{p}=0.99$. Results are shown for four different turbulence levels. The dotted line shows the result for amplitude parameter $\eta=0$ (no turbulence). The other curves represent, in order of increasing variability, $\eta=0.1,1$, and 10 . 
The general effect that turbulence has on mirroring is illustrated by the results of Experiments $1-3$, as shown in Figures 8 - 10. For these experiments, all particles were injected from the same location and with the same pitch angle, but the magnetic environments had different turbulence levels. Not surprisingly, the distributions broaden as the turbulence strength parameter $\eta$ increases. Interestingly, the distribution of mirroring radii for the $\eta=0.1$ and $\eta=1$ cases are well represented by normal distributions whose median values (dashed lines) are very nearly equal to the mirroring radius in the absence of turbulence (solid line). For the $\eta=10$ case, the distribution starts to deviate from normal and has a median value that is significantly greater than the turbulence-free mirroring radius. Note that for this latter case, the ratio of the turbulent to underlying field magnitudes at the location of the turbulent free mirror point scales as $\delta B / B_{0} \sim \sqrt{\eta} / \bar{\xi}_{m 0} \sim 0.2$, suggesting that "strong" turbulence enhances the mirroring effect, thereby reducing the ability of charged particles to penetrate into regions of increasing magnetic fields. In contrast, the corresponding ratios for the $\eta=0.1$ and $\eta=1.0$ cases are $\delta B / B_{0} \sim 0.02$ and $\delta B / B_{0} \sim 0.07$, respectively.

The results of Experiments 2, 4 and 5 illustrate how reflection is affected by the field line on which a particle moves. As shown by Figures 9, 11 and 12, there is little difference between the distributions of mirroring radii for particles spiraling inward along the $q=-9500$, $q=-5000$, and $q=0$ field lines. We note, also, that the turbulence-free mirror points along different field lines converge as $\xi \rightarrow 1$, as illustrated in Figure 5. As a result, there is no need to weight the distributions of initial positions when considering the overall effects of funneling and mirroring; this finding validates our use of random starting positions along the $x-y$ plane for injection scenarios 2 and 3 , as defined above.

How turbulence affects mirroring for particles able to penetrate further into the field as a result of a smaller injection pitch angle is illustrated by the results of Experiments $6-8$, each of which adopts injection scenario 2. Consistent with the results of Experiments $1-3$, turbulence is seen to enhance mirroring in the "strong" turbulence limit. Indeed, the ratios of the turbulent to underlying field magnitudes at the location of the turbulent-free mirror point for Experiments $6-8$ are given by $\delta B / B_{0} \sim 0.07, \delta B / B_{0} \sim 0.02$ and $\delta B / B_{0} \sim 0.7$ respectively. We also note that the distributions obtained for both Experiments 7 and 8 are quite similar, and are both characterized by median values of $\bar{\xi}_{m} \approx 8$. This result suggests that once a certain turbulence threshold $\delta B / B_{0} \sim 1 / 8$ is reached, particles are effectively mirrored.

Experiments 9 - 13 explore how effective turbulence is likely to be at limiting the number of cosmic rays reaching the star/disk system. Particle injection scheme 3 is adopted, for which half the particles are injected with a small enough pitch angle to reach the inner boundary in the absence of turbulence, as indicated by the distribution shown in Figure 16. 
As is clearly seen from Figures 17 - 20, the presence of turbulence significantly reduces the number of cosmic rays that reach the star/disk system, though cosmic rays with a smaller energy do seem to be more likely to do so. In addition, Kraichnan turbulence $(\Gamma=3 / 2)$ appears to be slightly more effective than Kolmogorov tubulence $(\Gamma=5 / 3)$ at limiting the number of cosmic rays that reach the star/disk system. This result is consistent with the fact that Kraichnan turbulence has more power at shorter wavelengths, and therefore can more effectively scatter lower energy particles. Finally, we note, that the ratios of the turbulent to underlying field magnitudes were mirroring occurs (as characterized by the median of the distribution), is $\delta B / B_{0} \sim 0.1, \delta B / B_{0} \sim 0.1, \delta B / B_{0} \sim 0.2$, and $\delta B / B_{0} \sim 0.1$ for Experiments $10,11,12$ and 13 , respectively.

\section{Conclusion}

This paper considers the effects of turbulent fluctuations on the propagation of cosmic rays impinging upon young star/disk systems. We focus on the case of magnetic fields with hour-glass-like configurations and show how turbulence influences the magnetic mirroring of incoming cosmic rays. The most important effects of turbulence are to replace the mirroring point with a distribution of values and to move the median mirror point outwards for sufficiently large fluctuation amplitudes. More specifically, our results can be summarized as follows:

We first construct a new coordinate system such that one coordinate follows the magnetic field lines of the hour-glass configuration (Section 2). The perpendicular coordinate is then used to construct Alfvénic field fluctuations, i.e., perturbations that are perpendicular to the original magnetic field lines (Section 3). Using the divergence operator of the new coordinate system, we can ensure that the perturbations are divergence-free.

Using this formulation of the problem, we have performed a large number of numerical integrations for cosmic rays propagating along the magnetic field lines, including the turbulent fluctuations. The relevant parameter space is large: One must consider the field line in question (labeled by its coordinate value $q$ ), the relative strength $\varepsilon$ of the split-monopole and background field contributions, the relative strength $\eta$ of the fluctuating field components compared to the unperturbed field, as well as the initial energy (given by the the Lorentz factors $\gamma$ ) and injection inclination angle $\alpha_{p}$ of the cosmic rays. In addition, for each choice of the variables $\left(q, \varepsilon, \eta, \gamma, \alpha_{p}\right)$, cosmic rays will experience different realizations of the turbulent

fluctuations. As a result, an ensemble of integrations must be carried out for each set of starting conditions. 
Table 1. Summary of Experiments

\begin{tabular}{rcccccccc}
\hline \hline $\operatorname{Exp}$ & IS & $\eta$ & $\Gamma$ & $\gamma$ & $\cos \alpha_{p}$ & $q$ & $\bar{\xi}_{m} / \bar{\xi}_{m 0}$ & $\sigma_{\xi} / \bar{\xi}_{m}$ \\
\hline 1 & 1 & 0.1 & $5 / 3$ & $10^{2}$ & 0.99 & -5000 & 1.0 & 0.08 \\
2 & 1 & 1 & $5 / 3$ & $10^{2}$ & 0.99 & -5000 & 1.0 & 0.26 \\
3 & 1 & 10 & $5 / 3$ & $10^{2}$ & 0.99 & -5000 & 1.2 & 0.41 \\
4 & 1 & 1 & $5 / 3$ & $10^{2}$ & 0.99 & -9500 & 1.0 & 0.29 \\
5 & 1 & 1 & $5 / 3$ & $10^{2}$ & 0.99 & 0 & 1.0 & 0.23 \\
6 & 2 & 1 & $5 / 3$ & $10^{2}$ & 0.99 & - & 1.0 & 0.28 \\
7 & 2 & 1 & $5 / 3$ & $10^{2}$ & 0.999 & - & 1.8 & 0.41 \\
8 & 2 & 1 & $5 / 3$ & $10^{2}$ & 0.9999 & - & 5.8 & 0.36 \\
9 & 3 & 0 & $5 / 3$ & $10^{2}$ & - & - & 1.0 & - \\
10 & 3 & 0.1 & $5 / 3$ & $10^{2}$ & - & - & 3.1 & - \\
11 & 3 & 1 & $5 / 3$ & $10^{2}$ & - & - & 8.2 & - \\
12 & 3 & 1 & $5 / 3$ & 10 & - & - & 5.8 & - \\
13 & 3 & 1 & $3 / 2$ & $10^{2}$ & - & - & 9.1 & -
\end{tabular}

Table 1: The columns give the values of the experiment number (Exp), the scheme for initial conditions (IS), the amplitude parameter for fluctuations $(\eta)$, the turbulence profile parameter $(\Gamma)$, the Lorentz factor $(\gamma)$, the starting injection angle $\left(\alpha_{p}\right)$, the coordinate that labels the field line $(q)$, the ratio of the median mirroring point to that obtained with no turbulence $\left(\bar{\xi}_{m} / \bar{\xi}_{m 0}\right)$, and finally the normalized width of the distribution of mirror point $\operatorname{radii}\left(\sigma_{\xi} / \bar{\xi}_{m}\right)$. 


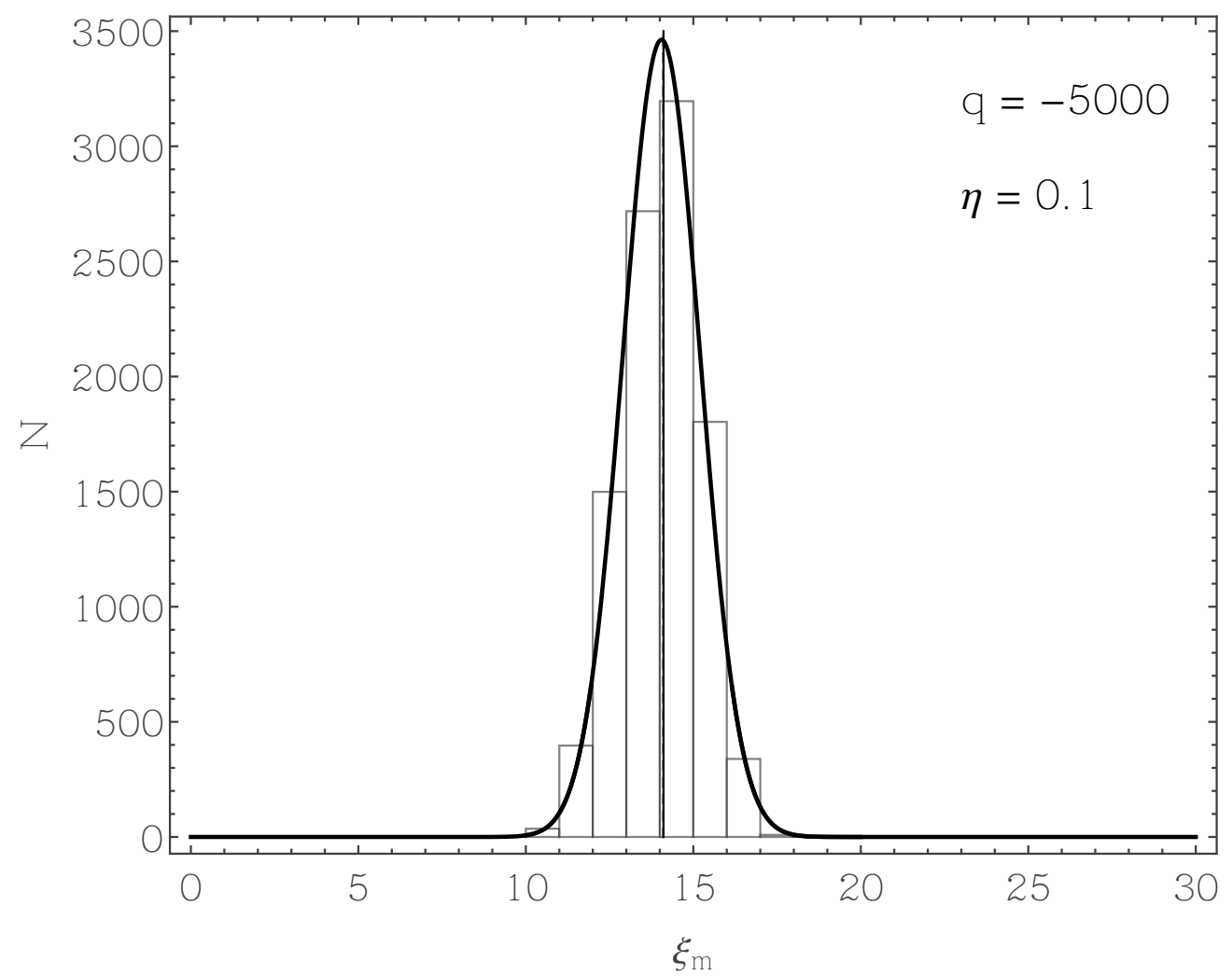

Fig. 8.- Result of Experiment 1, as presented through the distribution of mirror radii for $10^{3}$ particles injected along the $q=-5000$ field line, starting with $z_{i}=10^{3} R$, Lorentz factor $\gamma=10^{2}$, and with pitch angle $\cos \alpha_{p}=0.99$. The background field $B_{0}$ is defined through the parameter $\varepsilon=10^{4}$, and the turbulent magnetic field strength is set at $\eta=0.1$. The solid line denotes the value $\xi_{m 0}$ of the mirror radius in absence of turbulence, and the dashed line denotes the median value $\bar{\xi}_{m}$ of distribution. 


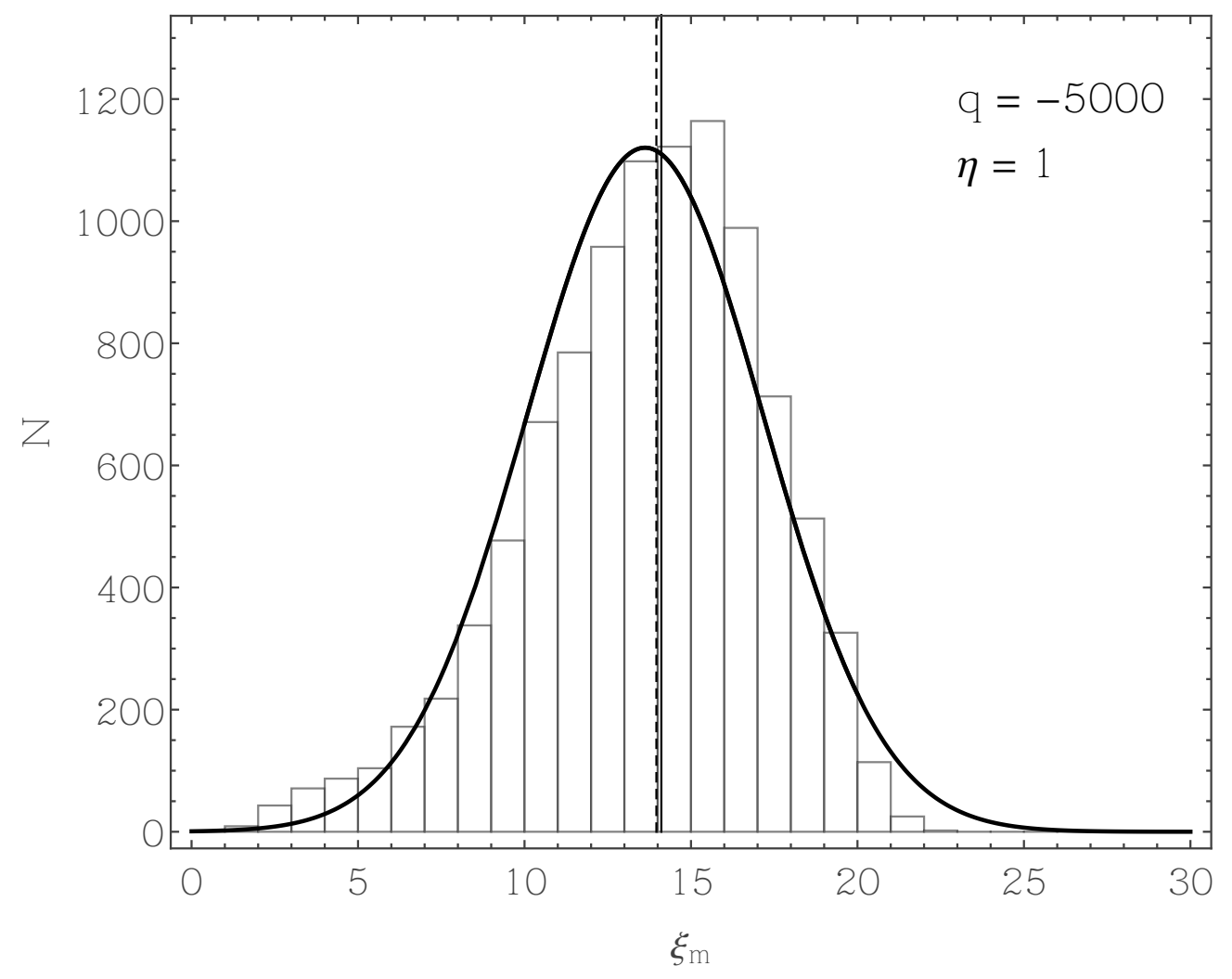

Fig. 9.- Same as Figure 8, but for Experiment 2, for which $\eta=1$. 


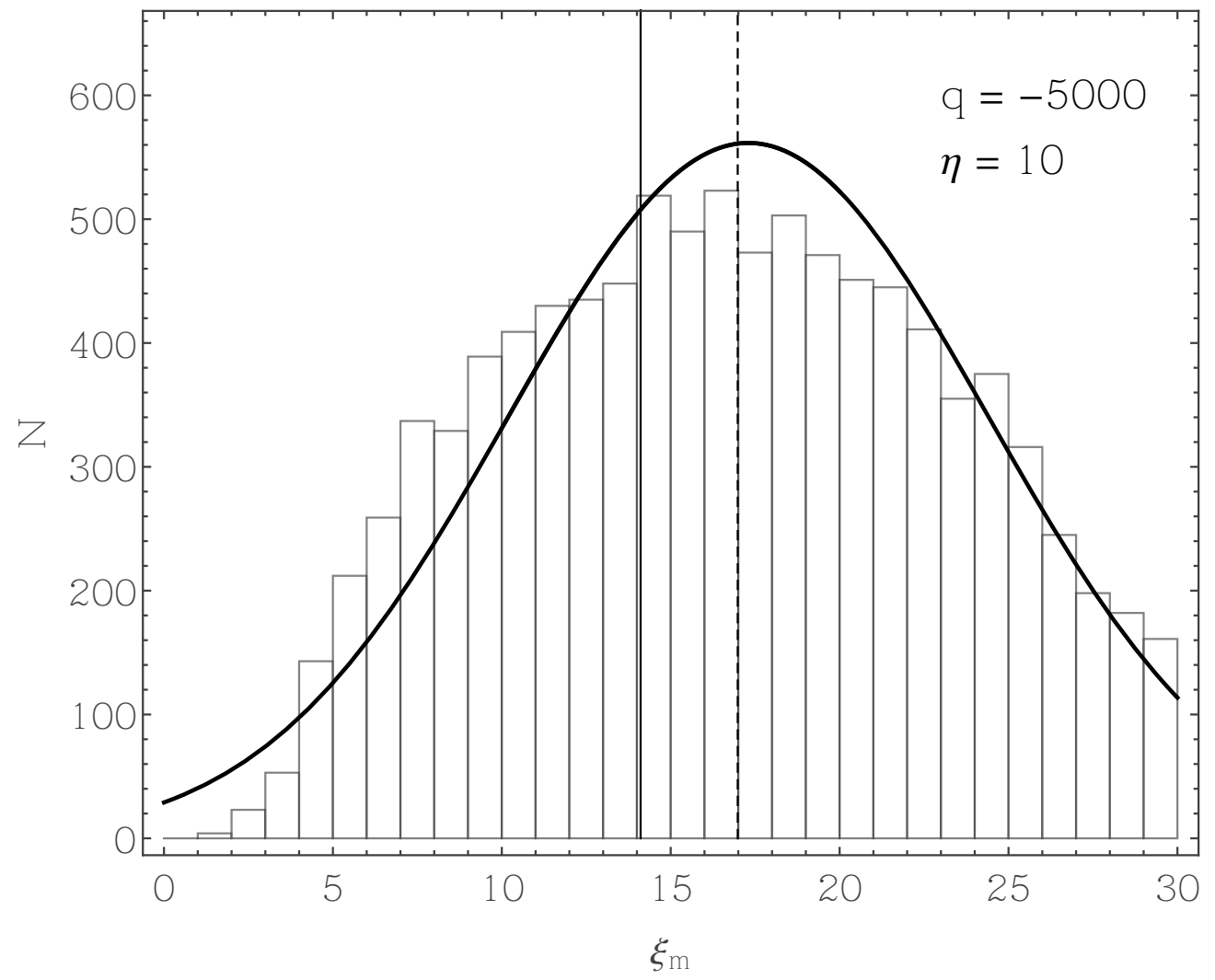

Fig. 10. - Same as Figure 8, but for Experiment 3, for which $\eta=10$. 


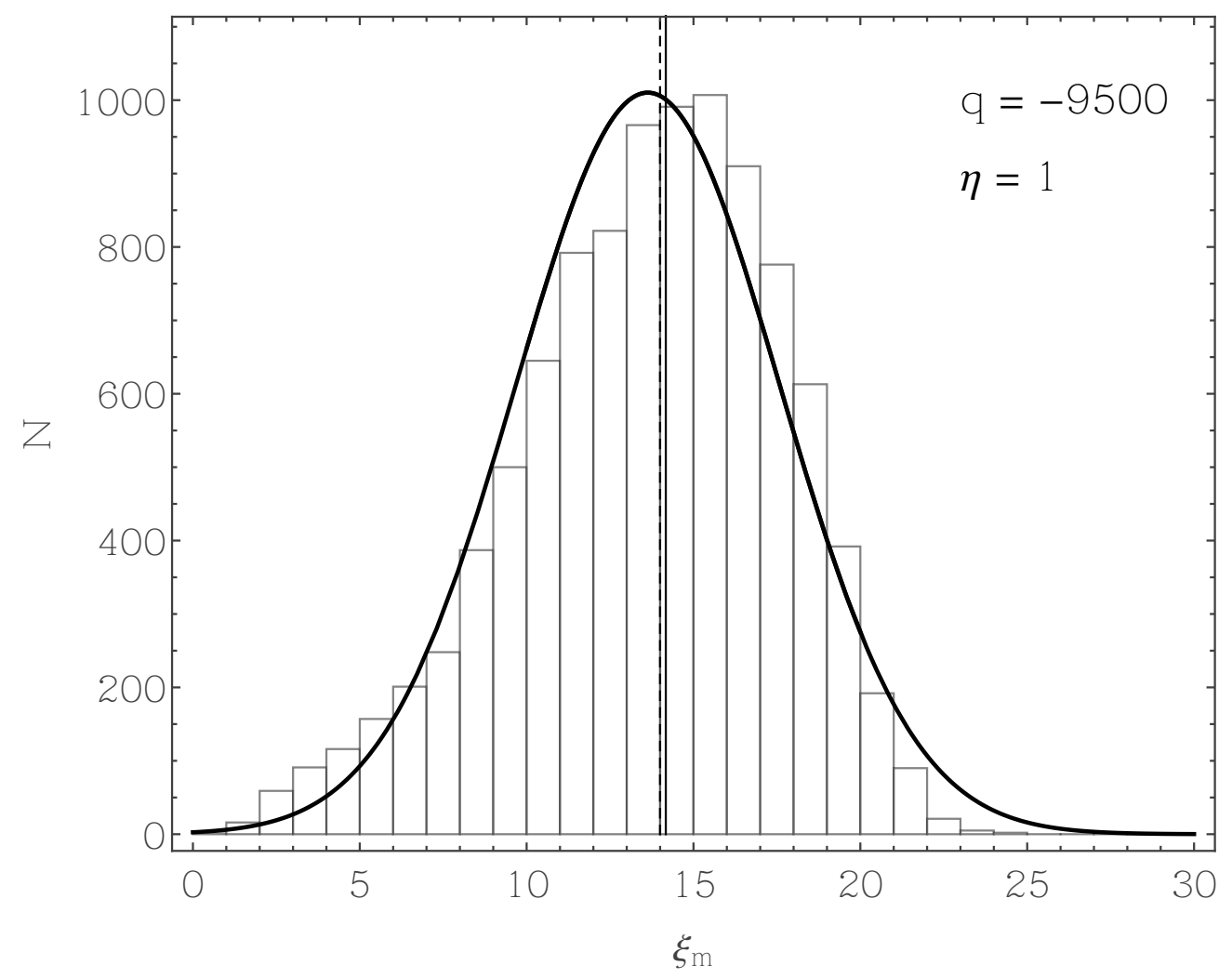

Fig. 11. - Same as Figure 8, but for Experiment 4, for which $\eta=1$ and $q=-9500$. 


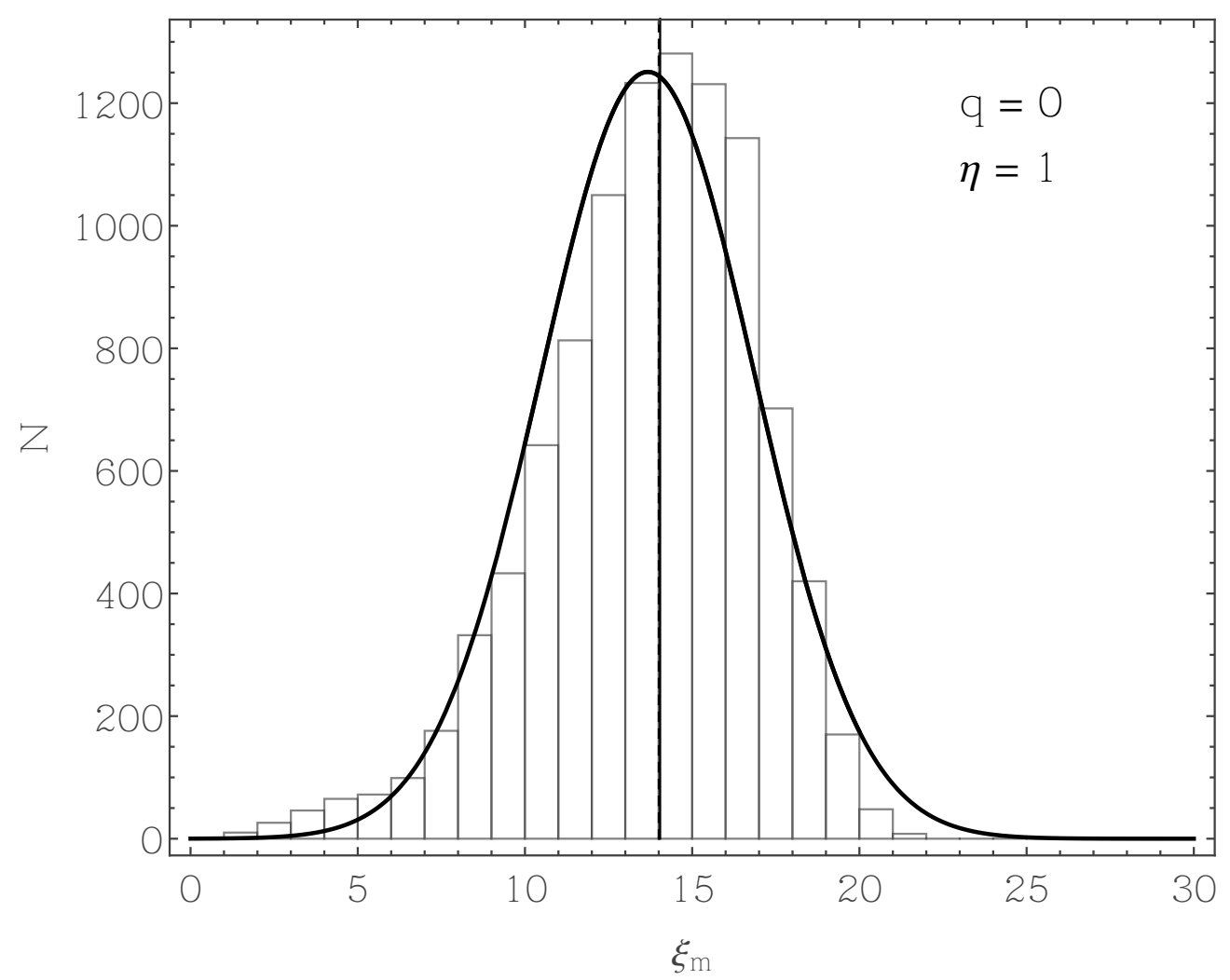

Fig. 12. - Same as Figure 8, but for Experiment 5, for which $\eta=1$ and $q=0$. 


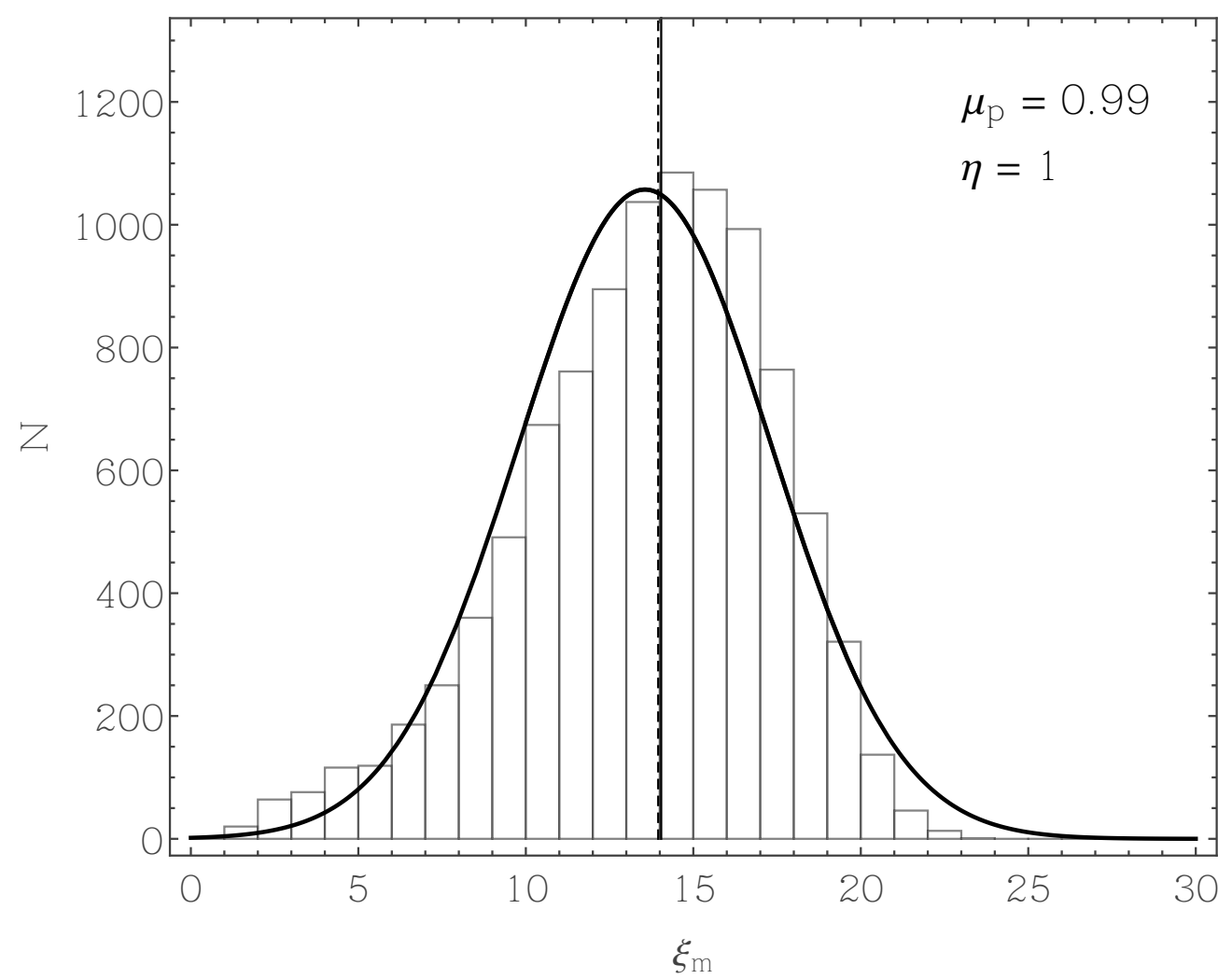

Fig. 13.- Result of Experiment 6, as presented through the distribution of mirror radii for $10^{3}$ particles with Lorentz factor $\gamma=10^{2}$ and injected with pitch angle $\cos \alpha_{p}=0.99$ from random locations at $z_{i}=10^{3} R$ from the part of $x-y$ plane that funnels particles directly onto the inner surface. The background field $B_{0}$ is defined through the parameter $\varepsilon=10^{4}$, and the turbulent magnetic field strength is set at $\eta=1$. The solid line denotes the value $\xi_{m 0}$ of the mirror radius in absence of turbulence, and the dashed line denotes the median value $\bar{\xi}_{m}$ of distribution. 


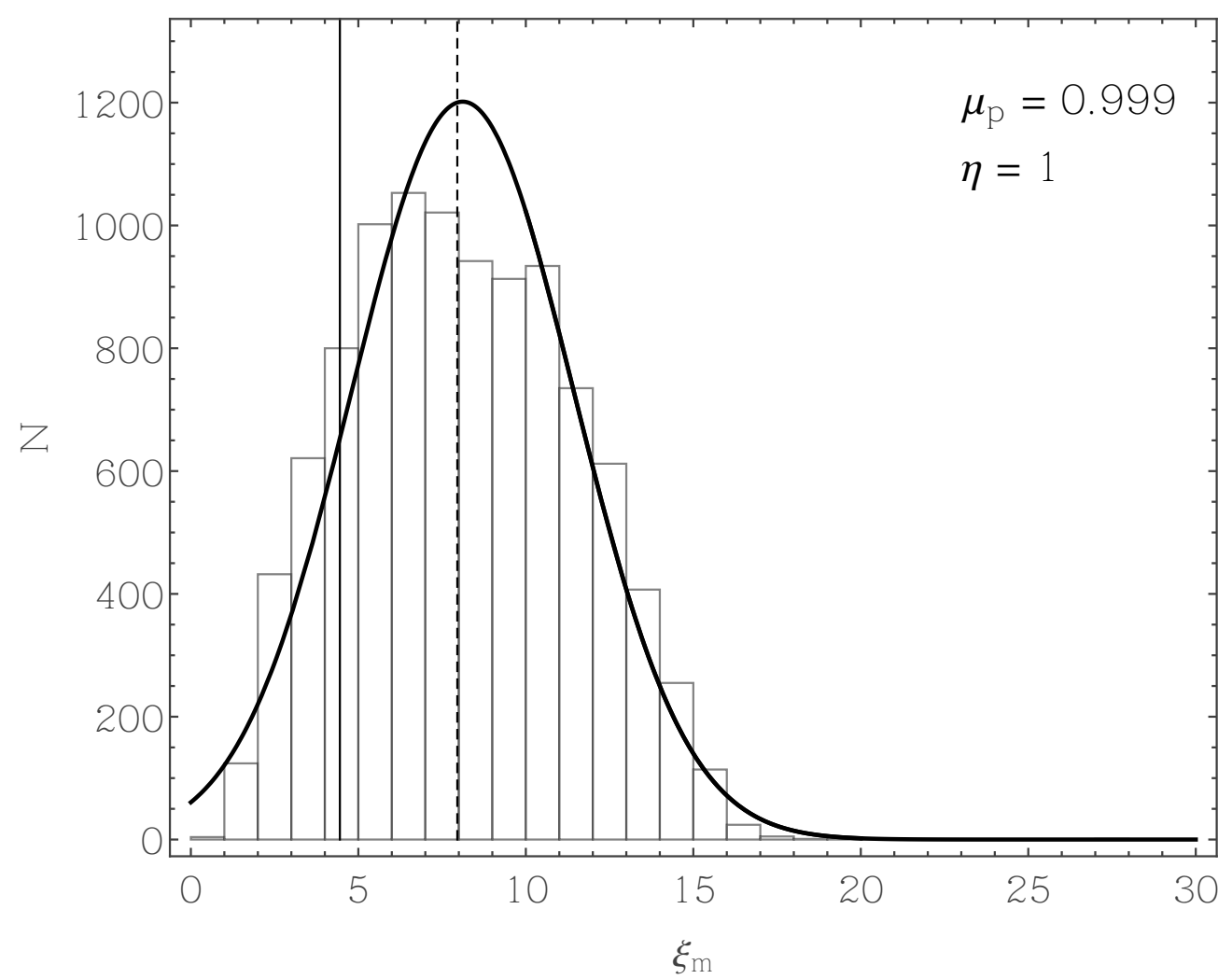

Fig. 14. - Same as Figure 13, but for Experiment 7, for which $\cos \alpha_{p}=0.999$. 


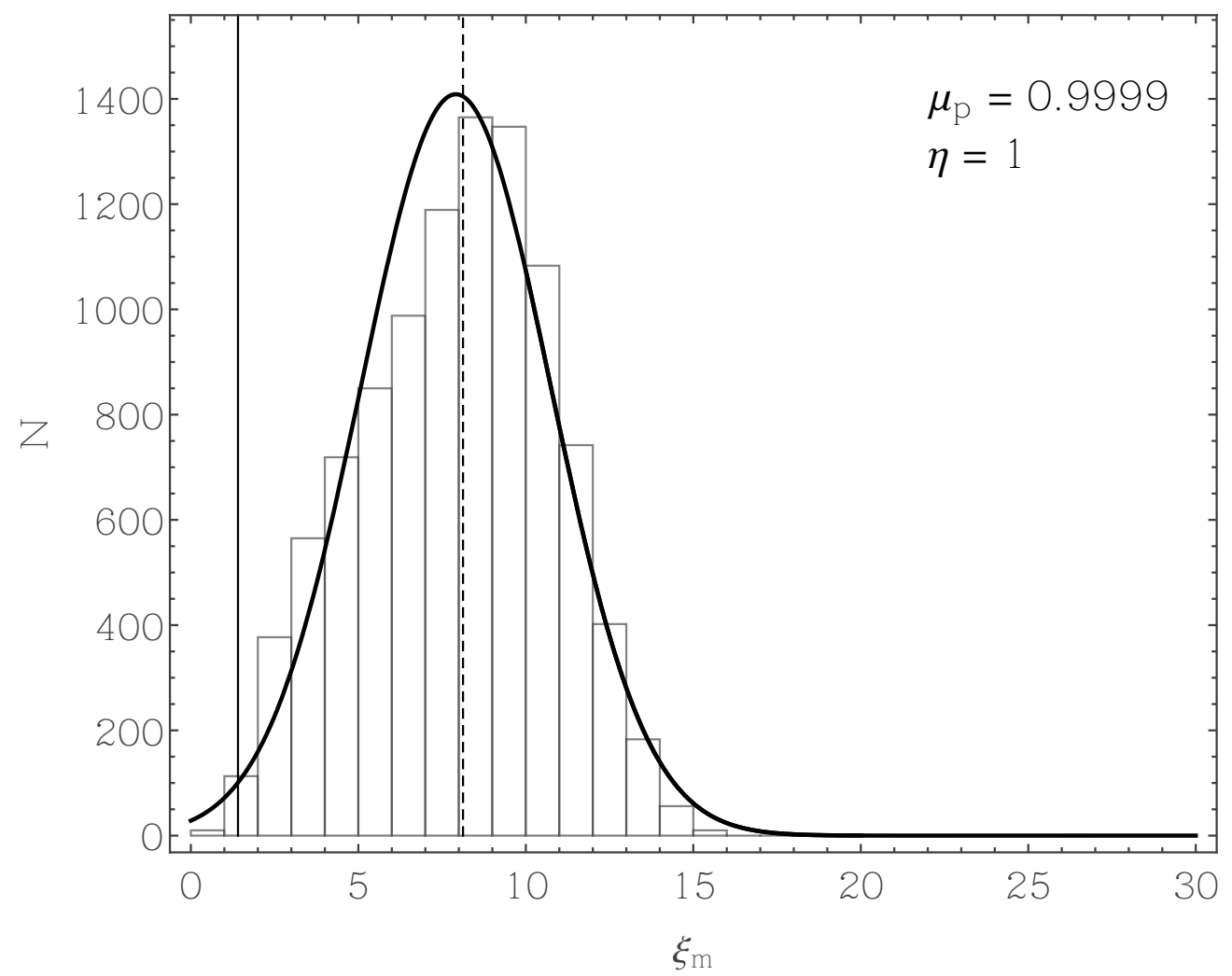

Fig. 15.- Same as Figure 13, but for Experiment 8, for which $\cos \alpha_{p}=0.9999$. 


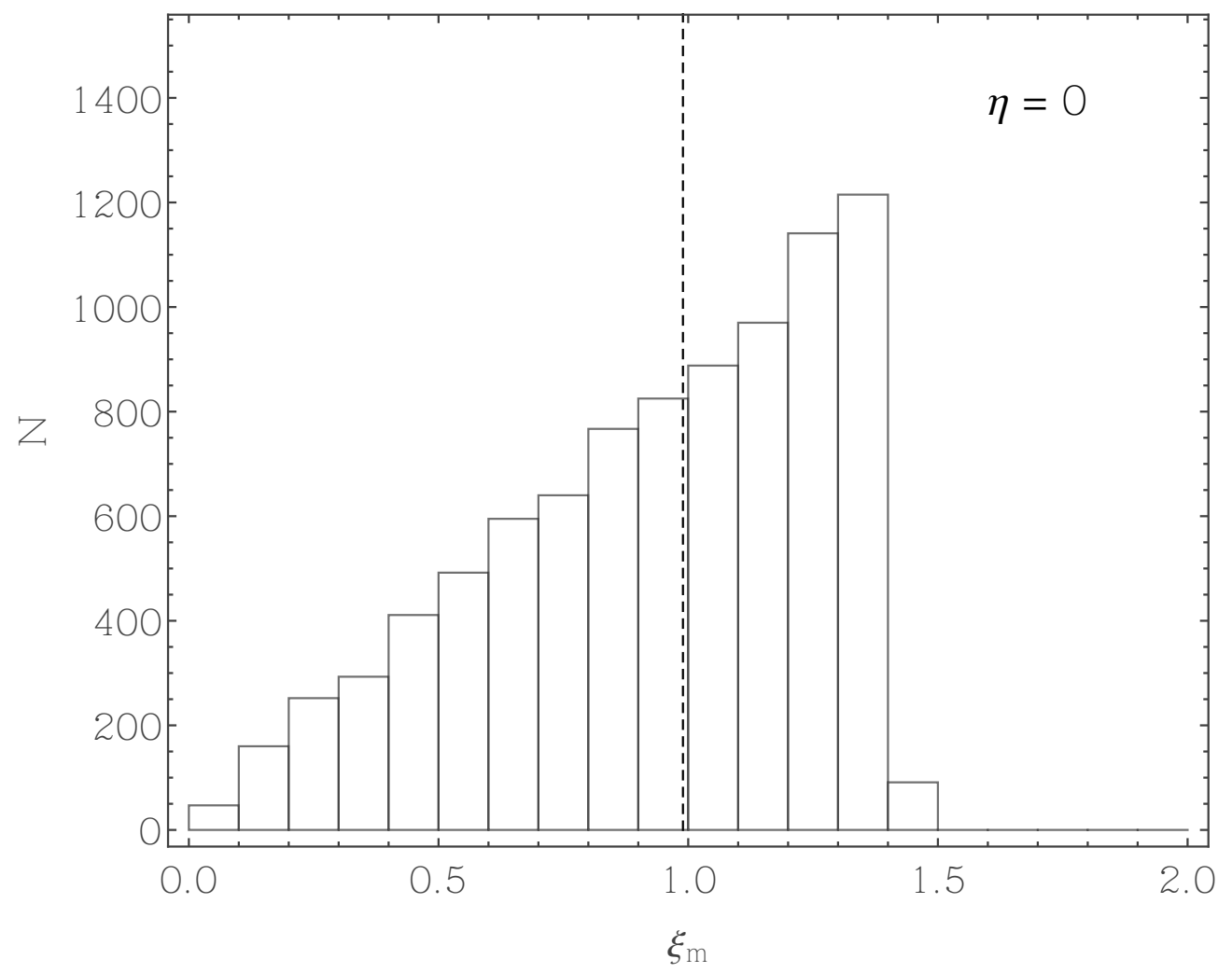

Fig. 16. - Result of Experiment 9, as presented through the distribution of mirror radii for $10^{3}$ particles with Lorentz factor $\gamma=10^{2}$ and injected with pitch angle randomly selected from a flat (uniform) distribution between $1 \geq \mu_{p} \geq 0.9999$, and from random locations at $z_{i}=10^{3} R$ from the part of $x-y$ plane that funnels particles directly onto the inner surface. The background field $B_{0}$ is defined through the parameter $\varepsilon=10^{4}$, and the turbulent magnetic field strength is set at $\eta=0$ (no turbulent field). The dashed line denotes the median value $\bar{\xi}_{m}$ of distribution. 


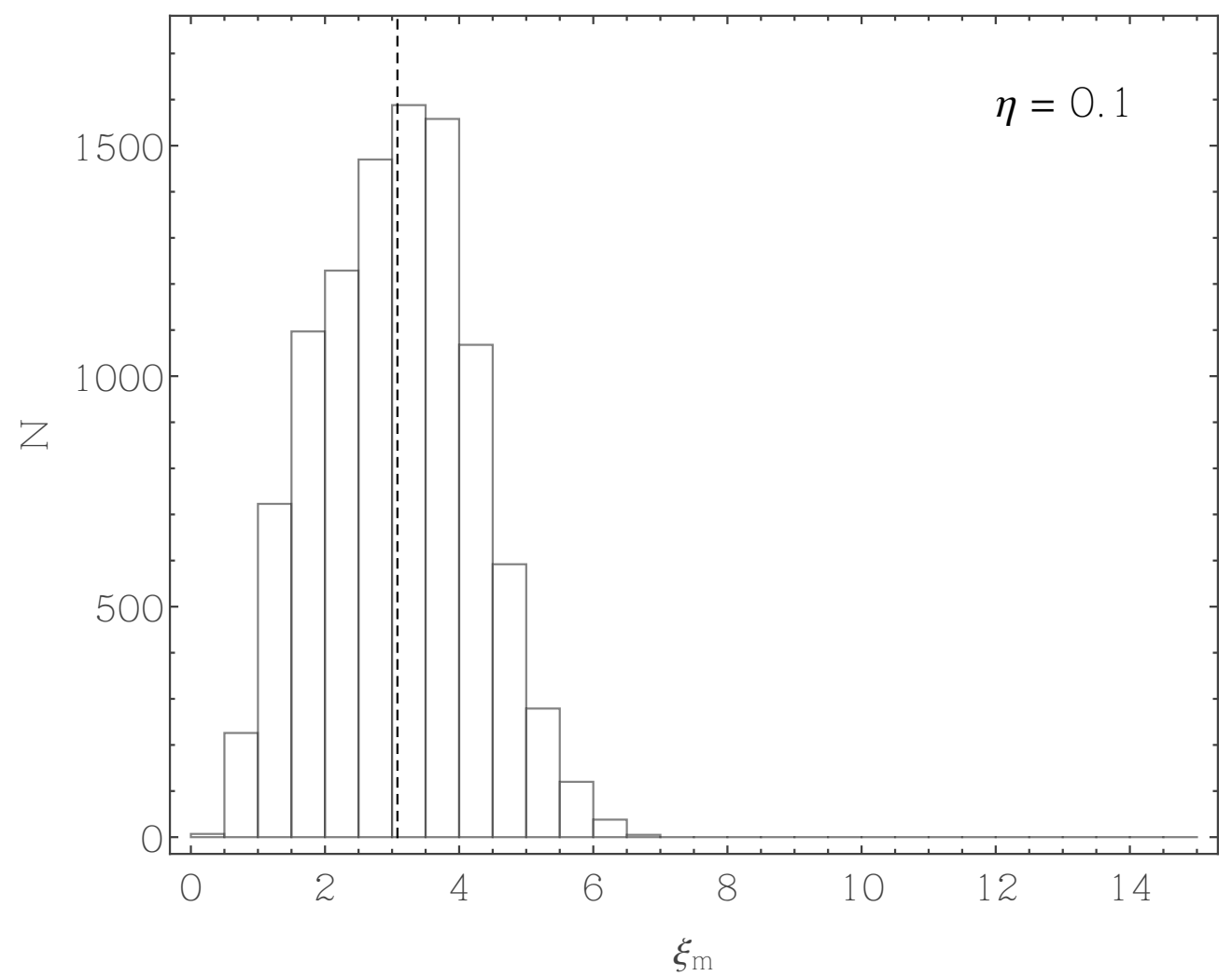

Fig. 17. - Same as Figure 16, but for Experiment 10, for which $\eta=0.1$. 


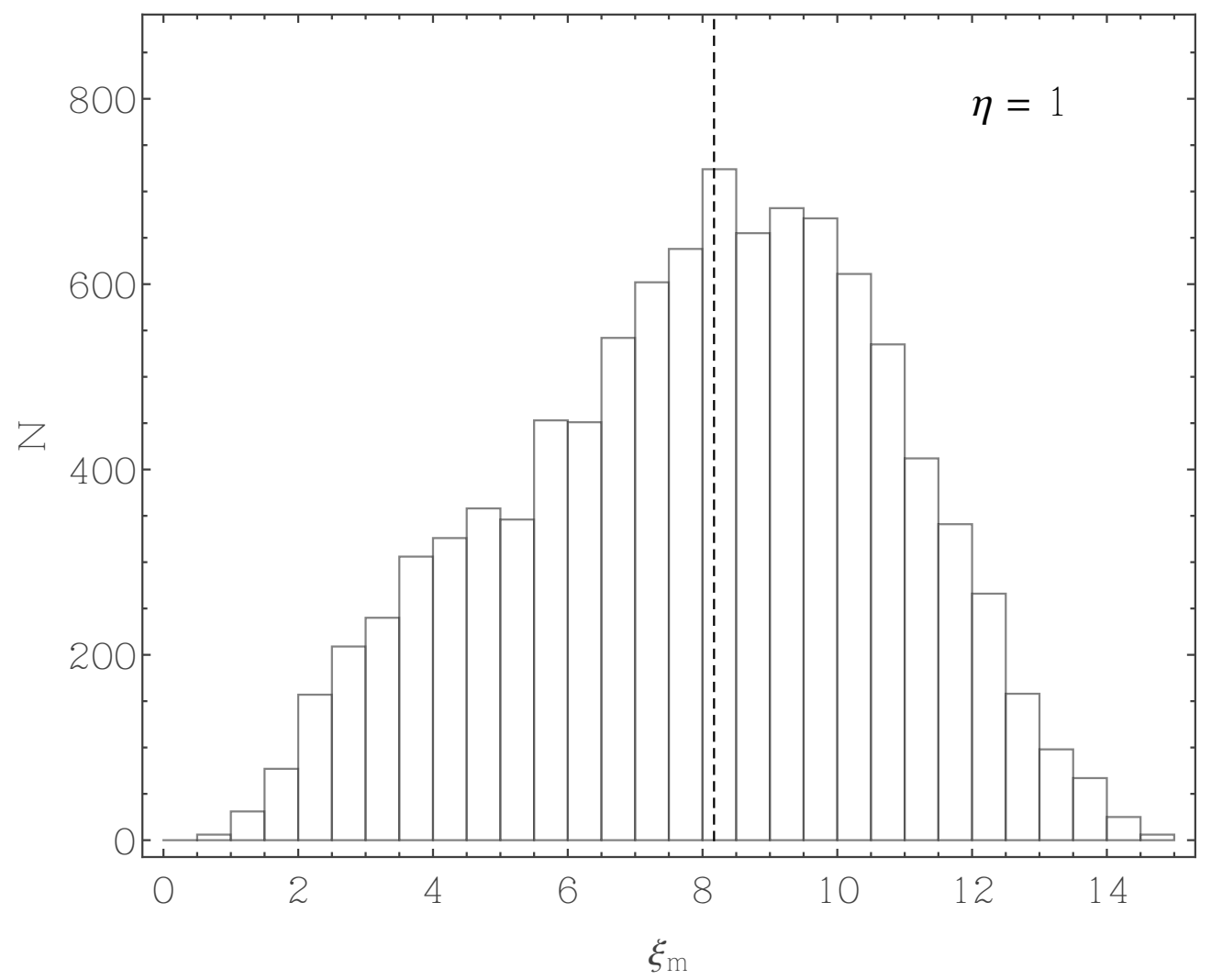

Fig. 18. - Same as Figure 16, but for Experiment 11, for which $\eta=1$. 


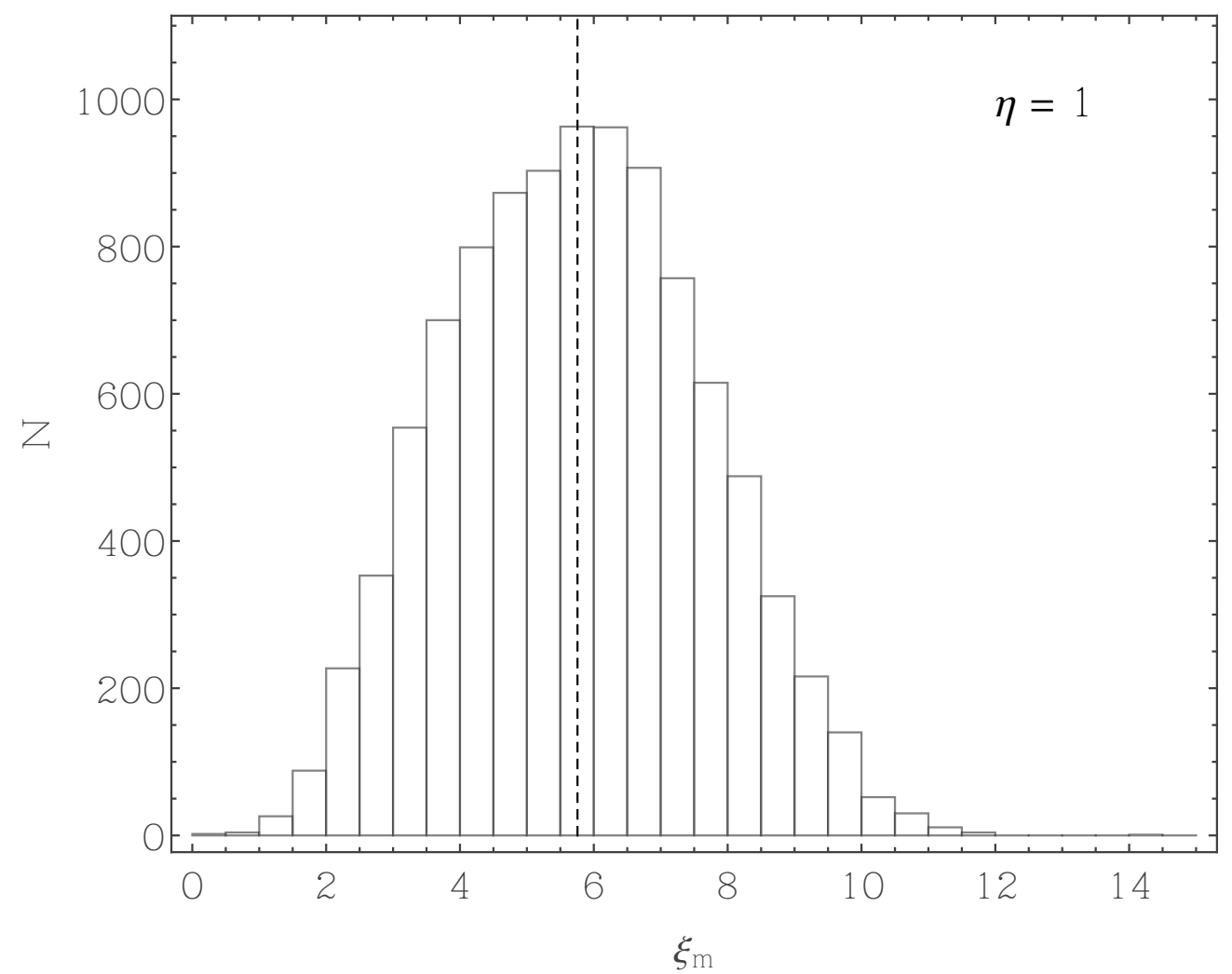

Fig. 19. - Same as Figure 16, but for Experiment 12, for which $\eta=1$ and $\gamma=10$. 


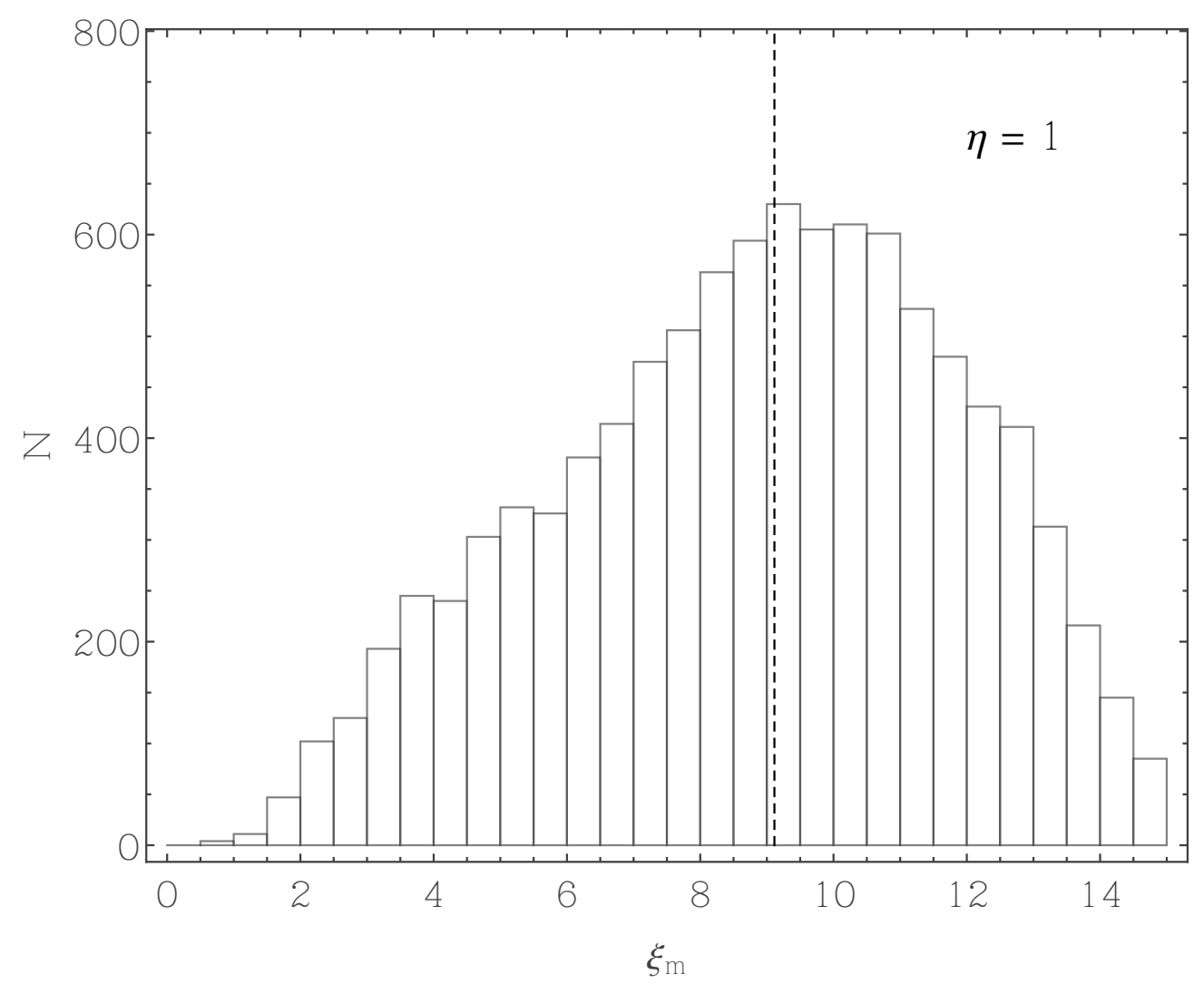

Fig. 20.- Same as Figure 16, but for Experiment 13, for which $\eta=1$ and $\Gamma=3 / 2$. 
The results of our numerical experiments (see Figures 8 - 19) provide us with the distribution of mirroring points for incoming cosmic rays for given sets of initial conditions. Most notable, turbulence affects the propagation of cosmic rays in these systems by replacing the mirror point with a distribution of values. If mirroring occurs at a location in the field for which the magnitude of the turbulent magnetic field component is small, $\delta B \ll 0.1 B_{0}$ (where $B_{0}$ is the magnitude of the underlying static field), the resulting distribution is well described by a normal distribution with a median value near the location of the mirror point found in the absence of turbulence. However, magnetic mirroring becomes enhanced once particles enter a regime with larger fluctuation amplitudes with $\delta B>0.1 B_{0}$, even though the particles could penetrate further into the turbulence-free field. The corresponding increase in the median mirror point radius can be large, up to an order of magnitude for the portion of parameter space considered herein (see Table 1). As a result, even a relatively modest amount of turbulence $(\eta \sim 0.1)$ in young stellar objects can significantly reduce the flux of cosmic rays reaching the disk.

A growing consensus in the field holds that disk accretion is produced by an effective viscosity that is driven by turbulence, which in turn is driven by MHD instabilities such as MRI (Balbus \& Hawley 1991). In order for MRI to operate, and hence for disk accretion to take place, the ionization fraction must be sufficiently high so that the gas is well coupled to the field. The inner disk can be ionized by collisions (where the number densities and temperatures are high), and the outer disk can be ionized by standard values of the cosmic ray flux, but intermediate regions may have dead zones where ionization is too low Gammie 1996). A reduced cosmic ray flux, such as that indicated here, will thus act to decrease the fraction of the disk that is active, i.e., sufficiently ionized for MRI to operate. For completeness we also note that $\mathrm{T}$ Tauri winds can also repel incoming cosmic rays, in analogy to the Solar wind (Cleeves et al. 2013a). As a result, the cosmic ray flux could be too low for the disk to be MRI active. In that case, the leading contribution to the ionization rate is given by the decay of short-lived radioactive nuclei (Umebayashi \& Nakano 2009, Cleeves et al. 2013b). An important topic for additional work is to ascertain how stellar winds and magnetic turbulence jointly modulate the incoming cosmic ray flux, and how the result compares to the contributions expected from radioactivity.

Another potential application of this work is to the magnetic braking catastrophe, which can occur during the earlier protostellar stage of evolution. In many circumstances, magnetic fields are so effective at removing angular momentum from infalling protostellar envelopes that circumstellar disks cannot form at all, or they are produced in highly truncated configurations (for further detail see, e.g., Li et al. (2013), along with references therein). The failure to produce disks is a theoretical problem, as observations indicate that circumstellar disks are ubiquitous around young stellar objects. If protostellar systems are sufficiently turbu- 
lent, however, magnetic field fluctuations can increase the efficiancy of mirroring and thereby reduce the cosmic ray flux in the inner region where disk formation takes place. With a lower cosmic ray flux, and hence lower ionization levels, the gas will be less well-coupled to the magnetic field, and magnetic braking can be compromised. Although turbulence acts in the right direction to alleviate the magnetic braking problem, further work must be carried out to determine the size of the effect. In particular, turbulence also acts to increase the rate of ambipolar diffusion (Fatuzzo \& Adams 2002; Zweibel 2002), and the rate of magnetic reconnection (Lazarian \& Vishniac 1999), and both of these processes remove magnetic fields from the inner collapse region and help facilitate disk formation. In addition, stochastic magnetic

reconnection in a partially ionized medium will also produce a magnetic cascade (Lazarian et al. 2004). An important challenge for the future is to understand the interplay between the reduction of ionization indicated here and the possible increased rates of magnetic field diffusion and reconnection.

Finally, we note that this paper assumes that turbulence remains robust in the inner regions of the hour-glass field configuration where magnetic mirroring takes place. This assumption could be modified by ion-neutral damping, which acts to reduce the amplitude of magnetic turbulence when the frequency of magnetic waves is of the order of, or larger than, the ion-neutral collisional frequency. If ion-neutral damping is sufficiently effective, the turbulence levels in the inner regions could be lower than assumed here. The degree of ionneutral damping depends on the magnetic field strength, the ionization levels, the density of the background gas, and other parameters, all of which have significant uncertainties, and all of which vary within the inner regime of the young stellar object. As a result, the level of ion-neutral damping, and the corresponding amplitudes for the turbulence, are uncertain. Adding to the uncertainty, additional sources of turbulence (e.g., from protostellar winds and outflows) could also be operative. This paper parameterizes the degree of turbulence through the parameter $\eta$ (see Table 1). Another challenge for the future is to develop a self-consistent model for the turbulence for this inner region.

This work was supported by Xavier University through the Hauck Foundation. The authors thank the referee for useful comments that improved the manuscript.

\section{REFERENCES}

Adams, F. C. 2011, ApJ, 730, 27 
Adams, F. C., \& Gregory, S. G. 2012, ApJ, 744, 55

Balbus, S. A., \& Hawley, J. F. 1991, ApJ, 376, 214

Casse, F., Lemoine, M., \& Pelletier, G. 2002, Phys. Rev. D, 65, 023002

Cesarsky, C. J., \& Völk, H. J. 1978, A\&A, 70, 367

Chandran, B.D.G. 2000, ApJ, 529, 513

Chapman, N. L., et al. 2013, ApJ, 770, 151

Cho, J., \& Lazarian, A. 2003, MNRAS, 345, 325

Cleeves, L. I., Adams, F. C., \& Bergin. E. A. 2013, ApJ, 772, 5

Cleeves, L. I., Adams, F. C., Bergin. E. A., \& Visser, R. 2013, ApJ, 777, 28

Crutcher, R. M. 2012, ARA\&A, 50, 29

Davidson, J. A., Novak, G., Matthews, T. G., Matthews, B., Goldsmith, P. F., Chapman, N., Volgenau, N. H., Vaillancourt, J. E., \& Attard, M. 2011, ApJ, 732, 97

Dalgarno, A. 2006, Publ. Nat. Acad. Sci., 103, 12269

De Marco, D., Blasi, P., \& Todor, S. 2007, JCAP, 6, 27

Desch, S. J., Connolly, H. C., Jr., \& Srinivasan, G. 2004, ApJ, 602, 528

Everett, J. E., \& Zweibel, E. G. 2011, ApJ, 739, 60

Fatuzzo, M., \& Adams, F. C. 2002, ApJ, 570, 210

Fatuzzo, M., Adams, F. C., \& Melia, F. 2006, ApJ, 653, L49

Fatuzzo, M., Melia, F., Todd, E., \& Adams, F. C. 2010, ApJ, 725, 515

Gammie, C. F. 1996, ApJ, 457, 355

Giacalone, J. \& Jokipii, J. R. 1994, ApJL, 430, L137

Glassgold, A. E., \& Langer, W. D. 1973, ApJ, 186, 859

Goldreich, P., \& Sridhar, S. 1995, ApJ, 438, 763

Hayakawa, S., Nishimura, S., \& Takayanagi, T. 1961, PASJ, 13, 184 
Jokipii, J. R. 1966, ApJ, 146, 480

Lazarian, A., \& Vishniac, E. T. 1999, ApJ, 517, 700

Lazarian, A., Vishniac, E. T., \& Cho, 2004, ApJ, 603, 180

Li, Z.-Y., Kransnopolsky, R., \& Shang, H. 2013, ApJ, 774, 82

McKee, C. F., \& Ostriker, E. C. 2007, ARA\&A, 45, 565

O’Sullivan, S., Reville, B., \& Taylor, A. M. 2009, MNRAS, 400, 248

Padoan, P., \& Scalo, J. 2005, ApJ, 624, L97

Padovani, M., \& Galli, D. 2011, A\&A, 530, 109

Padovani, M., Hennebelle, P., \& Galli, D. 2013, A\&A, 560, 114

Shu, F. H., Adams, F. C., \& Lizano, S. 1987, ARA\&A, 25, 23

Skilling, J., \& Strong, A. W. 1976, A\&A, 53, 253

Spitzer, L., \& Tomasko, M. G. 1968, ApJ, 152, 971

Sridhar, S., \& Goldreich, P. 1994, ApJ, 432, 612

Umebayashi, T., \& Nakano, T. 2009, ApJ, 690, 69

Zweibel, E. 2002, ApJ, 567, 962 